\title{
A Complete Axiomatisation for Divergence Preserving Branching Congruence of Finite-State Behaviours
}

\author{
Xinxin Liu \\ State key laboratory of Computer Science, ISCAS \\ University of Chinese Academy of Sciences \\ RISE, School of Computer and Information Science \\ South West University, China \\ Email: xinxin@ios.ac.cn
}

\author{
Tingting Yu \\ Beijing Sunwise information Technology Ltd \\ Beijing Institute of Control Engineering \\ Email: yutingting@sunwiseinfo.com
}

\begin{abstract}
We present an equational inference system for finite-state expressions, and prove that the system is sound and complete with respect to divergence preserving branching congruence, closing a problem that has been open since 1993. The inference system refines Rob van Glabbeek's simple and elegant complete axiomatisation for branching bisimulation congruence of finite-state behaviours by joining four simple axioms after dropping one axiom which is unsound under the more refined divergence sensitive semantics.
\end{abstract}

\section{INTRODUCTION}

Over the years the notion of bisimulation which was proposed by Park and popularized by the work of Milner emerges as a very important foundation for concurrency theory. Based on this notion, many interesting equivalence and congruence relations are introduced and studied. Rob van Glabbeek gave a fairly complete list of these equivalences in [5] and [6].

For a bisimulation based congruence relation on a set of expressions, an interesting question is whether there is an equational inference system, or axiomatisation, which infers exactly the pairs of equal expressions. Even in the cases where the equivalence relation is decidable, an inference system of this nature is still important since it conveys valuable information about the rationale behind the equalities. For finite-state expressions (expressions which can only generate finitely many states but may generate infinite behaviours), Milner pioneered this line of research, and proposed complete axiomatisations for strong bisimulation congruence [1] and observational bisimulation congruence [2]. Following Milner's work, Walker [4] and Lohrey et al. [13] proposed complete axiomatisations for variations of observational bisimulation congruence which take divergence behaviour into account, and van Glabbeek proposed a complete axiomatisation for branching bisimulation congruence [11].

The notion of branching bisimulation was introduced by van Glabbeek and Weijland in [10], in which a refined notion of divergence preservation is introduced and used to define

978-1-6654-4895-6/21/\$31.00 @2021 IEEE divergence-preserving version of the corresponding bisimulation equivalence and congruence. So, in [11] van Glabbeek posed the following natural question: to find a complete axiomatisation for the divergence-preserving version of branching bisimulation congruence. The problem remains open until today, although Chen and $\mathrm{Lu}[14]$ and $\mathrm{Fu}[16]$ proposed complete axiomatisations for divergence-preserving semantics for sub-languages of finite-state behaviours. In this paper, we propose an axiomatisation for finite-state behaviours, and prove its soundness and completeness with respect to divergencepreserving branching bisimulation congruence.

The divergence-preserving branching bisimulation equivalence with the corresponding congruence is unique in that it is the finest possible bisimulation equivalence which abstracts from internal moves. Thus, a complete axiomatisation for the congruence could serve as a core theory which can be readily extended to axiomatisations for other bisimulation based congruences by adding new axioms.

For the completeness proof, we use Milner's [1] framework of set of guarded equations, with the following difference: instead of using the product construction of equation sets proposed by Milner, we use the quotient construction which was introduced by Grabmayer and Fokkink in [19] and independently by Liu and $\mathrm{Yu}$ in [20].

The paper is organized as follows. In the next section we settle the preliminaries, including the definitions and properties of the equivalence and congruence relations. In section III we present the inference system and prove its soundness for divergence-preserving branching congruence. In section IV we introduce the notion of standard sum, and prove a standardization result: every expression can be proven equal to a standard sum. In section $\mathrm{V}$ we study standard equation systems (SES), and prove the quotient theorem, i.e. equivalent formal variables of an SES have common provable solution in a related guarded equation system. Using the result of section $\mathrm{V}$, the completeness of the inference system is proved in section VI. Then, we conclude in section VII. 


\section{Expressions, Divergence-PrESERVING SEMANTICS}

Let $\mathcal{V}$ be an infinite set of variables, $\mathcal{A}$ be an infinite set of visible actions, $\tau$ be the invisible action or silent move $(\tau \notin \mathcal{A})$. We write $\mathcal{A}_{\tau}$ for $\mathcal{A} \cup\{\tau\}$. Consider the set $\mathcal{E}$ of process expressions, given by the following BNF rules:

$$
E \quad::=0|X| a . E|E+E| \mu X . E
$$

where $a \in \mathcal{A}_{\tau}, X \in \mathcal{V}$. The precise meaning of the expressions will be given by operational semantics later. Here we provide the following explanations for the syntax, which may help to understand the intuitive meaning of the expressions:

- $\mathbf{0}$ is the expression which is not capable of any action;

- $a . E$ is a prefix expression which first performs the action $a$ and then proceeds as $E$;

- $E+F$ is a non-deterministic expression which is capable of actions from $E$ and $F$;

- $\mu X . E$ is a recursion which behaves as $E$ except that whenever $X$ is encountered in an execution then the rest behaviour is as $\mu X$.E.

We assume the usual notion of free and bound occurrence of variables with respect to the variable binder $\mu$, write $F V(E)$ for the set of free variables of $E$, and write $E\{F / X\}$ for the resulting expression obtained by (capture free) substitution of $F$ for (free occurrences of) $X$ in $E$. For a set of variables $\left\{X_{1}, \ldots, X_{n}\right\}$, we write $E\left\{F_{1} / X_{1}, \ldots, F_{n} / X_{n}\right\}$ for the simultaneous (capture free) substitution of $F_{1}$ for $X_{1}, \ldots$, $F_{n}$ for $X_{n}$ in $E$. Sometimes we will also use set notation to write simultaneous substitution of $F_{i}$ for $X_{i}$ in $E$ for each $i \in I$ as $E\left\{F_{i} / X_{i} \mid i \in I\right\}$, where $I$ is an index set. $\left\{F_{i} / X_{i} \mid i \in I\right\}$ can also be used standing alone to represent the intended substitution. Note that $E\left\{E_{1} / X_{1}\right\}\left\{E_{2} / X_{2}\right\}$ stands for the expression obtained by successive substitution of $E_{1}$ for the free occurrences of $X_{1}$ in $E$, and then $E_{2}$ for the free occurrences of $X_{2}$ in $E\left\{E_{1} / X_{1}\right\}$. We write $E \equiv F$ when $E, F$ are syntactically identical expressions.

The operational semantics of expressions is given by a transition relation $\longrightarrow$ and a binary relation $\triangleright$ between expressions and variables defined as follows.

Definition 2.1: The transition relation $\longrightarrow \subseteq \mathcal{E} \times \mathcal{A}_{\tau} \times \mathcal{E}$ is the smallest relation such that (we write $E \stackrel{a}{\longrightarrow} E^{\prime}$ for $\left.\left(E, a, E^{\prime}\right) \in \longrightarrow\right)$ :

1) $a . E \stackrel{a}{\longrightarrow} E$;

2) If $E_{1} \stackrel{a}{\longrightarrow} E^{\prime}$ then $E_{1}+E_{2} \stackrel{a}{\longrightarrow} E^{\prime}$;

3) If $E_{2} \stackrel{a}{\longrightarrow} E^{\prime}$ then $E_{1}+E_{2} \stackrel{a}{\longrightarrow} E^{\prime}$;

4) If $E\{\mu X . E / X\} \stackrel{a}{\longrightarrow} E^{\prime}$ then $\mu X . E \stackrel{a}{\longrightarrow} E^{\prime}$.

The relation $\triangleright \subseteq \mathcal{E} \times \mathcal{V}$ is the smallest relation such that (we write $E \triangleright X$ for $(E, X) \in \triangleright)$ :

1) $X \triangleright X$;

2) If $E_{1} \triangleright X$ then $E_{1}+E_{2} \triangleright X$;

3) If $E_{2} \triangleright X$ then $E_{1}+E_{2} \triangleright X$;

4) If $E\{\mu Y . E / X\} \triangleright X$ then $\mu Y . E \triangleright X$.

Also we write $\Longrightarrow$ for $(\stackrel{\tau}{\longrightarrow})$ * and $\tilde{\triangleright}$ for $\Longrightarrow \triangleright$ (in this paper we write $R^{*}$ for the reflexive and transitive closure of a binary relation $R \subseteq \mathcal{E} \times \mathcal{E}$, and write $R_{1} R_{2}$ for the composition of binary relations $R_{1}$ and $R_{2}$ when $R_{1}$ 's codomain and $R_{2}$ 's domain are both $\mathcal{E}$ ).

In an expression $E$, an occurrence of a variable $X$ is said guarded if the occurrence is within a subexpression $a . E^{\prime}$ of $E$ where $a \neq \tau$. $X$ is said guarded in $E$ if every free occurrence of $X$ in $E$ is a guarded occurrence.

To see some examples, $\tau . X+a .0 \tilde{\triangleright} X$, and if $X \notin F V(E)$ and $a \neq \tau$, then $X$ is guarded in $\tau$. $(E+a .(F+\tau . X))$.

For $E \in \mathcal{E}$, an infinite $\tau$-run from $E$ is an infinite sequence of expressions $E_{0} E_{1} \ldots E_{i} \ldots$ such that $E_{0} \equiv E$ and $E_{i-1} \stackrel{\tau}{\longrightarrow} E_{i}$ for $i>0$.

Next we state some lemmas which are needed later.

Lemma 2.2: Let $E, F, H \in \mathcal{E}, a \in \mathcal{A}_{\tau}, X \in \mathcal{V}$. Then:

1) If $H\{E / X\} \stackrel{a}{\longrightarrow} F$, then either there is $H^{\prime}$ such that $H \stackrel{a}{\longrightarrow} H^{\prime}$ and $F \equiv H^{\prime}\{E / X\}$, or $H \triangleright X$ and $E \stackrel{a}{\longrightarrow} F$;

2) If $H\{E / X\} \triangleright Y$, then either $H \triangleright Y$, or $H \triangleright X$ and $E \triangleright Y$

3) If $H \stackrel{a}{\longrightarrow} H^{\prime}$, then $H\{E / X\} \stackrel{a}{\longrightarrow} H^{\prime}\{E / X\}$;

4) If $H \triangleright X$ and $E \stackrel{a}{\longrightarrow} F$ then $H\{E / X\} \stackrel{a}{\longrightarrow} F$.

The proof of this lemma can be found in [11] (Lemma 4).

Lemma 2.3: Let $X$ be a variable, $H \in \mathcal{E}$. Then $X$ occurs unguarded in $H$ if and only if $H \tilde{\triangleright} X$.

Proof It is easy to prove by induction on the rules defining $\longrightarrow$ and $\triangleright$ the following:

1) if $H \triangleright X$ then $X$ occurs unguarded in $H$;

2) if $H \stackrel{\tau}{\longrightarrow} H^{\prime}$ and $X$ occurs unguarded in $H^{\prime}$ then $X$ occurs unguarded in $H$.

Note that $H \tilde{\triangleright} X$ is $H \Longrightarrow \triangleright X$. Then the "if" direction can be proved by an easy induction on the length of transition sequence $\Longrightarrow$, and the "only if" direction can be proved by using Lemma 2.2 and analyzing the structure of $H$.

Lemma 2.4: Let $E, F \in \mathcal{E}, a \in \mathcal{A}_{\tau}, X, W \in \mathcal{V}$.

1) $\mu X . E \stackrel{a}{\longrightarrow} F$ if and only if there is $E^{\prime} \in \mathcal{E}$ such that $E \stackrel{a}{\longrightarrow} E^{\prime}$ and $F \equiv E^{\prime}\{\mu X . E / X\}$.

2) $\mu X . E \triangleright W$ if and only if $E \triangleright W$ and $W, X$ are different variables.

Proof. The proof of 1) can be found in [13] (Lemma 6). The proof of 2) is straightforward.

To present the definition of divergence-preserving branching bisimulation, we first define a number of functions on binary relations, and study the relationships of these functions.

Definition 2.5: For a binary relation $R \subseteq \mathcal{E} \times \mathcal{E}$, define binary relations $\mathcal{S}(R), \mathcal{B}(R), \mathcal{B}^{\prime}(R)$, and $\mathcal{B}^{\triangle}(R)$ as follows:

1) $\mathcal{S}(R)$ is a binary relation such that $(E, F) \in \mathcal{S}(R)$ if:

a) whenever $E \stackrel{a}{\longrightarrow} E^{\prime}$, then there exists $F^{\prime}$ such that $F \stackrel{a}{\longrightarrow} F^{\prime}$ and $\left(E^{\prime}, F^{\prime}\right) \in R$;

b) whenever $E \triangleright X$, then $F \triangleright X$.

2) $\mathcal{B}(R)$ is a binary relation such that $(E, F) \in \mathcal{B}(R)$ if:

a) whenever $E \stackrel{a}{\longrightarrow} E^{\prime}$, then either $a=\tau$ and there exists $F^{\prime}$ s.t. $F \Longrightarrow F^{\prime}$ and $\left(E, F^{\prime}\right),\left(E^{\prime}, F^{\prime}\right) \in R$, or there exist $F^{\prime}, F^{\prime \prime}$ s.t. $F \Longrightarrow F^{\prime}, F^{\prime} \stackrel{a}{\longrightarrow} F^{\prime \prime}$ and $\left(E, F^{\prime}\right),\left(E^{\prime}, F^{\prime \prime}\right) \in R$;

b) whenever $E \triangleright X$, then there exists $F^{\prime}$ such that $F \Longrightarrow F^{\prime},\left(E, F^{\prime}\right) \in R$, and $F^{\prime} \triangleright X$. 
3) $\mathcal{B}^{\prime}(R)$ is a binary relation such that $(E, F) \in \mathcal{B}^{\prime}(R)$ if:

a) whenever $E \stackrel{a}{\longrightarrow} E^{\prime}$, then either $a=\tau$ and there exists $F^{\prime}$ s.t. $F \stackrel{\tau}{\longrightarrow} \Longrightarrow F^{\prime}$ and $\left(E, F^{\prime}\right),\left(E^{\prime}, F^{\prime}\right) \in R$, or there exist $F^{\prime}, F^{\prime \prime}$ s.t. $F \Longrightarrow F^{\prime}, F^{\prime} \stackrel{a}{\longrightarrow} F^{\prime \prime}$ and $\left(E, F^{\prime}\right),\left(E^{\prime}, F^{\prime \prime}\right) \in R$;

b) whenever $E \triangleright X$, then there exists $F^{\prime}$ such that $F \Longrightarrow F^{\prime},\left(E, F^{\prime}\right) \in R$, and $F^{\prime} \triangleright X$.

4) $\mathcal{B}^{\triangle}(R)$ is a binary relation such that $(E, F) \in \mathcal{B}^{\triangle}(R)$ if:

a) $(E, F) \in \mathcal{B}(R)$;

b) whenever $E E_{1} \ldots E_{i} \ldots$ is an infinite $\tau$-run from $E$, then there exist $E_{j}$ on the $\tau$-run and $F^{\prime}$ such that $F \stackrel{\tau}{\longrightarrow} F^{\prime}$ and $\left(E_{j}, F^{\prime}\right) \in R$.

$\mathcal{S}, \mathcal{B}, \mathcal{B}^{\triangle}$ will be used to define corresponding bisimulation equivalences. $\mathcal{B}^{\prime}$ will be used in introducing a divergence preserving bisimulation verification technique. Note that $\mathcal{B}^{\prime}$ differs from $\mathcal{B}$ only in one place where $\mathcal{B}^{\prime}$ requires $\stackrel{\tau}{\longrightarrow} \Longrightarrow$ (in which at least one $\stackrel{\tau}{\longrightarrow}$ step must be made) instead of the transition sequence $\Longrightarrow$. The result of this subtle change made a big difference as $\mathcal{B}^{\prime}(R)$ becomes a subset of not only $\mathcal{B}(R)$ but also $\mathcal{B}^{\Delta}(R)$ for any binary relation $R$, as shown by the following lemma.

Lemma 2.6: Let $R \subseteq \mathcal{E} \times \mathcal{E}$. Then

$$
\mathcal{S}(R) \subseteq \mathcal{B}^{\prime}(R) \subseteq \mathcal{B}^{\triangle}(R) \subseteq \mathcal{B}(R) .
$$

Proof. $\mathcal{S}(R) \subseteq \mathcal{B}^{\prime}(R)$ and $\mathcal{B}^{\Delta}(R) \subseteq \mathcal{B}(R)$ can be checked immediately from the definitions. To see $\mathcal{B}^{\prime}(R) \subseteq \mathcal{B}^{\Delta}(R)$, note that from the definitions one easily observes $\mathcal{B}^{\prime}(R) \subseteq \mathcal{B}(R)$. Then for all $(E, F) \in \mathcal{B}^{\prime}(R)$, whenever $E E_{1} \ldots E_{i} \ldots$ is an infinite $\tau$-run from $E$, the transition $E \stackrel{\tau}{\longrightarrow} E_{1}$ would find some $F^{\prime}$ such that $F \stackrel{\tau}{\longrightarrow} \Longrightarrow F^{\prime}$ and $\left(E_{1}, F^{\prime}\right) \in R$, meeting the requirement of being a member of $\mathcal{B}^{\triangle}(R)$.

Definition 2.7: A binary relation $R \subseteq \mathcal{E} \times \mathcal{E}$ is a strong bisimulation if $R$ is symmetric and $R \subseteq \mathcal{S}(R)$.

A binary relation $R \subseteq \mathcal{E} \times \mathcal{E}$ is a branching bisimulation if $R$ is symmetric and $R \subseteq \mathcal{B}(R)$.

A binary relation $R \subseteq \mathcal{E} \times \mathcal{E}$ is a progressing branching bisimulation if $R$ is symmetric and $R \subseteq \mathcal{B}^{\prime}(R)$.

A binary relation $R \subseteq \mathcal{E} \times \mathcal{E}$ is a divergence-preserving branching bisimulation if $R$ is symmetric and $R \subseteq \mathcal{B}^{\Delta}(R)$.

Define three binary relations, called strong bisimilarity, branching bisimilarity, divergence-preserving branching bisimilarity, and written $\sim, \approx_{b}, \approx_{b}^{\Delta}$ respectively, as follows

$\sim=\bigcup\{R \mid R$ is a strong bisimulation $\}$,

$\approx_{b}=\bigcup\{R \mid R$ is a branching bisimulation $\}$,

$\approx_{b}^{\Delta}=\bigcup\{R \mid R$ is a div.-pre. branching bisimulatoin $\}$.

For $\sim, \approx_{b}, \approx_{b}^{\Delta}$, we have the following justification.

Theorem 2.8: $\sim, \approx_{b}$, and $\approx_{b}^{\Delta}$ are equivalence relations. Moreover

1) $\sim$ is the coarsest strong bisimulation;

2) $\approx_{b}$ is the coarsest branching bisimulation;

3) $\approx_{b} \Delta$ is the coarsest divergence-preserving branching bisimulation. Moreover, it is the coarsest equivalence relation which is a branching bisimulation and which preserves divergence, i.e. for two equivalent expressions if one has an infinite $\tau$-run within its equivalence class then so is the other.

Proof. 1), 2) are well known ([3], [10]). See [17] for 3).

Note that $\approx_{b}$ does not respect divergence. A simple example to show this is $\mu X .(\tau . X+a .0) \approx_{b} \tau . a .0$, where the left expression has an infinite $\tau$-run, while the right expression has not. Thus $\approx_{b}^{\Delta}$ provides an alternative when divergence needs to be taken into account. There are different presentations of divergence-preserving branching bisimilarity. It was called branching bisimilarity with explicit divergence in [10] and [15], and called complete branching bisimilarity in [17]. When first introduced in [10], it was defined as the coarsest equivalence relation which is a branching bisimulation and which preserves divergence. 3) of Theorem 2.8 shows that the present definition gives the same relation. In [15], a condition similar to condition b) in 4) of Definition 2.5 for matching a divergent run requires that $F^{\prime}$ is found after exactly one step of $\tau$ action. The condition b) in 4) of Definition 2.5] is from [17], which allows $F^{\prime}$ to be found after one or more steps of $\tau$ action. The discrepancy does not affect the resulting bisimilarity. One advantage of the weaker condition is a weaker divergence-preserving obligation in checking divergence-preserving branching bisimulation.

It is also worth noting that the divergence preserving condition for $\approx_{b}^{\Delta}$ is more strict than that required in the divergence preserving relations introduced earlier in [4] and [13], in that the earlier works does not concern about the different equivalence classes that passed through by a divergent run.

Lemma 2.9: A strong bisimulation is a progressing branching bisimulation, which in turn is a divergence-preserving branching bisimulation, which in turn is a branching bisimulation.

Proof. Immediately follows from Lemma 2.6 .

The idea of progressing branching bisimulation comes from the notion of progressing weak bisimulation in [8], where by applying the standard fixed-point definition as in Definition 2.7. the notion of progressing weak bisimulation results in an equivalence relation which is a congruence. While this is not the case for progressing branching bisimulation, i.e. progressing branching bisimulation equivalence defined in similar fashion is not a congruence. Here we refrain from introducing a new equivalence, but instead, with Lemma 2.9 . we can use progressing branching bisimulation as a tool to establish divergence-preserving branching bisimulation, and it turned out to suit this role very well.

Proposition 2.10: $\subseteq \approx_{b}^{\Delta} \subseteq \approx_{b}$.

Proof. Immediately follows from Lemma 2.9

Proposition 2.11: Let $I d_{\mathcal{E}}=\{(E, E) \mid E \in \mathcal{E}\}$. Then $\mathcal{B}\left(I d_{\mathcal{E}}\right)$ is a divergence-preserving branching bisimulation.

Proof. Easy to check.

Proposition 2.11 can be strengthened to state that $\mathcal{B}\left(\approx_{b}^{\Delta}\right)$ is a divergence-preserving branching bisimulation, which can be a very useful technique in establishing divergence-preserving branching bisimulation, since the troublesome condition about 
infinite $\tau$-run in $\mathcal{B}^{\Delta}$ is avoided. However Proposition 2.11 is sufficient for the following development.

Lemma 2.12: Let $E, F \in \mathcal{E}$. Then $\tau .(E+F)+F \approx_{b}^{\Delta} E+F$. Proof. It is easy to verify that $(\tau .(E+F)+F, E+F) \in \mathcal{B}\left(I d_{\mathcal{E}}\right)$. By Proposition 2.11 $\mathcal{B}\left(I d_{\mathcal{E}}\right)$ is a divergence-preserving branching bisimulation, thus $\tau$. $(E+F)+F \approx_{b}^{\Delta} E+F$ follows from Definition 2.7

Lemma 2.13: Let $E, F \in \mathcal{E}, X \in \mathcal{V}$. If $E \approx_{b}^{\Delta} F$ then $E \tilde{\triangleright} X$ if and only if $F \tilde{\triangleright} X$.

Proof. Straightforward from Definition 2.5 and 2.7

Divergence-preserving branching bisimilarity $\approx_{b}^{\Delta}$ is not a congruence on $\mathcal{E}$. For a simplest counter example, note that and $a .0 \approx_{b}^{\Delta} \tau . a .0$ while $a .0+b .0 \approx_{b}^{\Delta} \tau . a .0+b .0$ when $a, b$ are different non- $\tau$ actions. This is solved in [18] by adding a rootedness condition.

Definition 2.14: Two expressions $E$ and $F$ are rooted divergence-preserving branching bisimilar, notation $E={ }_{b}^{\Delta} F$, if the following hold:

1) whenever $E \stackrel{a}{\longrightarrow} E^{\prime}$ then $F \stackrel{a}{\longrightarrow} F^{\prime}$ with $E^{\prime} \approx_{b}^{\Delta} F^{\prime}$;

2) whenever $F \stackrel{a}{\longrightarrow} F^{\prime}$ then $E \stackrel{a}{\longrightarrow} E^{\prime}$ with $E^{\prime} \approx_{b}^{\Delta} F^{\prime}$;

3) $E \triangleright X$ if and only if $F \triangleright X$.

Proposition 2.15: $\sim \subseteq={ }_{b}^{\Delta}$.

Proof. Immediately follows from the definitions.

The following theorem shows that $=_{b} \Delta$ is a congruence relation, thus from now on we call it divergence-preserving branching congruence.

Theorem 2.16: ${ }_{b}^{\Delta}$ is a congruence on $\mathcal{E}$, i.e. if $E={ }_{b}^{\Delta} F$ then $a \cdot E={ }_{b}^{\Delta} a \cdot F, E+D={ }_{b}^{\Delta} F+D, D+E={ }_{b}^{\Delta} D+F$, and $\mu X . E={ }_{b}^{\Delta} \mu X . F$ for arbitrary $a \in \mathcal{A}_{\tau}, D \in \mathcal{E}, X \in \mathcal{V}$.

Proof. Only $\mu X . E={ }_{b}^{\Delta} \mu X . F$ needs a proof $\left(E={ }_{b}^{\Delta} F\right.$ assumed), all the rest are easy. The divergence-preserving nature of the relation made it much harder to prove than expected. A detailed proof is presented in [18].

Rob van Glabbeek et al. also proved in [18] that $=_{b}^{\Delta}$ is the weakest congruence that implies divergence-preserving branching bisimilarity.

We close this section by introducing two versions of the very useful up-to technique. The notion of strong bisimulation up to $\sim$ is well known $([3])$, while that of strong bisimulation up to $\approx_{b}^{\Delta}$ is new.

Definition 2.17: A binary relation $R \subseteq \mathcal{E} \times \mathcal{E}$ is a strong bisimulation up to $\sim$ if it is symmetric and $R \subseteq \mathcal{S}(\sim R \sim)$.

A binary relation $R \subseteq \mathcal{E} \times \mathcal{E}$ is a strong bisimulation up-to $\approx_{b}^{\Delta}$ if it is symmetric and moreover the following hold for all $(E, F) \in R$ :

1) whenever $E \stackrel{\tau}{\longrightarrow} E^{\prime}$ then there exists $F^{\prime}$ such that $F \stackrel{\tau}{\longrightarrow} F^{\prime}$ and $\left(E^{\prime}, F^{\prime}\right) \in R$;

2) whenever $E \stackrel{a}{\longrightarrow} E^{\prime}$ for $a \neq \tau$ then there exists $F^{\prime}$ such that $F \stackrel{a}{\longrightarrow} F^{\prime}$ and $\left(E^{\prime}, F^{\prime}\right) \in \approx_{b}^{\Delta} R \approx_{b}^{\Delta}$;

3) whenever $E \triangleright X$ then $F \triangleright X$.

Lemma 2.18: Let $R \subseteq \mathcal{E} \times \mathcal{E}$.

1) If $R$ is a strong bisimulation up to $\sim$, then $R \subseteq \sim$.

2) If $R$ is a strong bisimulation up to $\approx_{b}^{\Delta}$, then $R \subseteq={ }_{b}^{\Delta}$.

Proof. 1) is proved in [3]. For 2), we first show that $\approx_{b}^{\Delta} R \approx_{b}^{\Delta}$ is a divergence-preserving branching bisimulation. Once this is done, then $R$ is a strong bisimulation up to $\approx_{b}^{\Delta}$ implies that $\approx_{b}^{\Delta} R \approx_{b}^{\Delta} \subseteq \approx_{b}^{\Delta}$. Then $R \subseteq={ }_{b}^{\Delta}$ immediately follows from the definition of strong bisimulation up to $\approx_{b}^{\Delta}$ and the definition of $={ }_{b}^{\Delta}$. In the rest of the proof we show that $\approx_{b}^{\Delta} R \approx_{b}^{\Delta}$ is a divergence-preserving branching bisimulation.

First it is easy to see that because $R$ is symmetric then so is $\approx_{b}^{\Delta} R \approx_{b}^{\Delta}$. We need the following simple property of $\approx_{b}^{\Delta}$ which is easy to establish: whenever $F \approx_{b}^{\Delta} G$ and $F \Longrightarrow F^{\prime}$ then there exists $G^{\prime}$ such that $G \Longrightarrow G^{\prime}$ and $F^{\prime} \approx_{b}^{\Delta} G^{\prime}$.

Suppose $E \approx_{b}^{\Delta} R \approx_{b}^{\Delta} F$, and $E \stackrel{a}{\longrightarrow} E^{\prime}$, we shall show that

(A) either $a=\tau$ and there exists $F^{\prime}$ such that $F \Longrightarrow F^{\prime}$, $E \approx_{b}^{\Delta} R \approx_{b}^{\Delta} F^{\prime}$ and $E^{\prime} \approx_{b}^{\Delta} R \approx_{b}^{\Delta} F^{\prime}$;

(B) or there exist $F^{\prime}, F^{\prime \prime}$ such that $F \Longrightarrow F^{\prime}, F^{\prime} \stackrel{a}{\longrightarrow} F^{\prime \prime}$, such that $E \approx_{b}^{\Delta} R \approx_{b}^{\Delta} F^{\prime}, E^{\prime} \approx_{b}^{\Delta} R \approx_{b}^{\Delta} F^{\prime \prime}$.

According to the meaning of relation composition, there exist $G, H \in \mathcal{E}$ such that $E \approx_{b}^{\Delta} G,(G, H) \in R$, and $H \approx_{b}^{\Delta} F$. By the branching bisimulation property of $\approx_{b}^{\Delta}$, for the transition $E \stackrel{a}{\longrightarrow} E^{\prime}$, either of the following must hold:

(a) $a=\tau$ and there exists $G^{\prime}$ such that $G \Longrightarrow G^{\prime}, E \approx_{b}^{\Delta} G^{\prime}$ and $E^{\prime} \approx_{b}^{\Delta} G^{\prime}$,

(b) there exist $G^{\prime}, G^{\prime \prime}$ such that $G \Longrightarrow G^{\prime}, G^{\prime} \stackrel{a}{\longrightarrow} G^{\prime \prime}$, such that $E \approx_{b}^{\Delta} G^{\prime}, E^{\prime} \approx_{b}^{\Delta} G^{\prime \prime}$.

We will show that (a) implies (A), and (b) implies either (A) or (B) to fulfill the above proof obligation.

If (a) is the case, from $G \Longrightarrow G^{\prime}$, according to case 1) in the definition of strong bisimulation up to $\approx_{b}^{\Delta}$, there exists $H^{\prime}$ such that $H \Longrightarrow H^{\prime}$ and $\left(G^{\prime}, H^{\prime}\right) \in R$, and then because $H \approx_{b}^{\Delta} F$, there exists $F^{\prime}$ such that $F \Longrightarrow F^{\prime}$ and $H^{\prime} \approx_{b}^{\Delta} F^{\prime}$. To summarize, in this case we find $F^{\prime}$, such that $F \Longrightarrow F^{\prime}$, and $E \approx_{b}^{\Delta} R \approx_{b}^{\Delta} F^{\prime}$, and $E^{\prime} \approx_{b}^{\Delta} R \approx_{b}^{\Delta} F^{\prime}$, that is to say (A) holds.

If (b) is the case, from $G \Longrightarrow G^{\prime}$ there exists $H^{\prime}$ such that $H \Longrightarrow H^{\prime}$ and $\left(G^{\prime}, H^{\prime}\right) \in R$, and since $R$ is a strong bisimulation up to $\approx_{b}^{\Delta}$, the transition $G^{\prime} \stackrel{a}{\longrightarrow} G^{\prime \prime}$ implies that there exists $H^{\prime \prime}$ such that $H^{\prime} \stackrel{a}{\longrightarrow} H^{\prime \prime}$ and $G^{\prime \prime} \approx_{b}^{\Delta} R \approx_{b}^{\Delta} H^{\prime \prime}$ (of cause it holds when $a=\tau$ ). Now because $H \approx_{b}^{\Delta} F$, from the move $H \Longrightarrow H^{\prime}$ there must exist $F_{0}$ such that $F \Longrightarrow F_{0}$ and $H^{\prime} \approx_{b}^{\Delta} F_{0}$. Now from $H^{\prime} \stackrel{a}{\longrightarrow} H^{\prime \prime}$, then since $H^{\prime} \approx_{b}^{\Delta} F_{0}$, either $a=\tau$ and there exists $F^{\prime}$ such that $F_{0} \Longrightarrow F^{\prime}$ and $H^{\prime} \approx_{b}^{\Delta} F^{\prime}$ and $H^{\prime \prime} \approx_{b}^{\Delta} F^{\prime}$, in this case we obtain $F \Longrightarrow F^{\prime}$ such that $E \approx_{b}^{\Delta} R \approx_{b}^{\Delta} F^{\prime}$ and $E^{\prime} \approx_{b}^{\Delta} R \approx_{b}^{\Delta} F^{\prime}$, that is to say (A) holds, or there exist $F^{\prime}, F^{\prime \prime}$ such that $F_{0} \Longrightarrow F^{\prime}$, $F^{\prime} \stackrel{a}{\longrightarrow} F^{\prime \prime}$ and $H^{\prime} \approx_{b}^{\Delta} F^{\prime}, H^{\prime \prime} \approx_{b}^{\Delta} F^{\prime \prime}$, in this case we obtain $F \Longrightarrow F^{\prime}$ and $F^{\prime} \stackrel{a}{\longrightarrow} F^{\prime \prime}$ such that $E \approx_{b}^{\Delta} R \approx_{b}^{\Delta} F^{\prime}$ and $E^{\prime} \approx_{b}^{\Delta} R \approx_{b}^{\Delta} F^{\prime \prime}$, that is to say (B) holds. To summarize, (b) implies either (A) or (B).

If $E \triangleright X$, in the same way we can show that there exists $F^{\prime}$ such that $F \Longrightarrow F^{\prime}, E \approx_{b}^{\Delta} R \approx_{b}^{\Delta} F^{\prime}$, and $F^{\prime} \triangleright X$.

Suppose $E E_{1} \ldots E_{i} \ldots$ is an infinite $\tau$-run, we have to show that there exists some $E_{k}$ on the infinite $\tau$-run and also exists $F^{\prime}$ such that $F \stackrel{\tau}{\longrightarrow} F^{\prime}$ and $E_{k} \approx_{b}^{\Delta} R \approx_{b}^{\Delta} F^{\prime}$.

In this case, again we can assume that there exist $G, H \in \mathcal{E}$ such that $E \approx_{b}^{\Delta} G,(G, H) \in R$, and $H \approx_{b}^{\Delta} F$. Then it is not difficult (could be lengthy though) to argue that there exists an infinite $\tau$-run $G G_{1} \ldots G_{j} \ldots$, such that for each $G_{j}$ on the run 
there exists $E_{i}$ on the $\tau$-run from $E$ with $E_{i} \approx_{b}^{\Delta} G_{j}$. Then by the definition of strong bisimulation up to $\approx_{b}^{\Delta}$ there exists an infinite $\tau$-run $H H_{1} \ldots H_{j} \ldots$ such that for each $H_{j}$ on the run, $G_{j}$ on the corresponding position of the $\tau$-run from $G$ satisfies $\left(G_{j}, H_{j}\right) \in R$. Then because $H \approx_{b}^{\Delta} F$, for this infinite $\tau$-run from $H$ there exists a $H_{k}$ on the $\tau$-run from $H$ and also exists $F^{\prime}$ such that $F \stackrel{\tau}{\longrightarrow} \Longrightarrow F^{\prime}$ and $H_{k} \approx_{b}^{\Delta} F^{\prime}$, and with $H_{k}$ we can find $G_{k}$ on the $\tau$-run from $G$ such that $\left(G_{k}, H_{k}\right) \in R$, and with $G_{k}$ we can find some $E_{i}$ on the $\tau$-run from $E$ such that $E_{i} \approx_{b}^{\Delta} G_{k}$. To summarize, we find $E_{i}$ on the $\tau$-run from $E$ and $F^{\prime}$ such that $F \stackrel{\tau}{\longrightarrow} \Longrightarrow F^{\prime}$ and $E_{i} \approx_{b}^{\Delta} R \approx_{b}^{\Delta} F^{\prime}$.

\section{The INFEREnCE System AND Its SOUNDNESS}

In this section we present our inference system for $=\underset{b}{\Delta}$ and prove its soundness. The following is the set of axioms and rules of the inference system, besides the rules for equational reasoning (reflexivity, symmetry, transitivity, and substituting equal for equal):

S1 $E+F=F+E$

$\mathrm{S} 2 E+(F+G)=(E+F)+G$

S3 $E+E=E$

$\mathrm{S} 4 \quad E+\mathbf{0}=E$

$\mathrm{B} a \cdot(\tau \cdot(E+F)+F)=a \cdot(E+F)$

R0 $\mu X . E=\mu Y .(E\{Y / X\})$

$\mathrm{R} 1 \mu X . E=E\{\mu X . E / X\}$

$(Y \notin F V(\mu X . E))$

R2 If $F=E\{F / X\}$ then $F=\mu X$.E, provided $X$ is guarded in $E$

R3 $\mu X .(X+E)=\mu X . E$

$\mathrm{R} 4 \mu X .(\tau .(\tau . E+F)+G)=\mu X .(\tau .(E+F)+G)$

R5 $\mu X .(\tau . \mu Y .(\tau . Y+E)+F)=\mu X .(\tau . \mu Y . E+F)$

$(E \tilde{\nabla} X)$

R6 $\mu X . \tau . E=\tau . \mu X . E\{\tau . X / X\}$

R7 $\mu X .(\tau . X+\mu Y .(\tau . Y+E))=\mu X . \mu Y(\tau . Y+E)$

R8 $\mu X . \mu Y .(\tau .(X+E)+F)=\mu X . \mu Y .(\tau .(Y+E)+F)$

We write $\vdash E=F$ if $E=F$ can be inferred from the above axioms and rules through equational reasoning. The aim of this section is to establish the soundness of the inference system with respect to $=_{b}^{\Delta}$, i.e. Theorem 3.5

S1-S4 are familiar axioms which appear in axiomatisations for all bisimulation based congruences. It is easy to show that S1-S4 are sound with respect to $\sim([3])$, hence are also sound with respect to $=_{b}^{\Delta}$ here by proposition 2.15.

$\mathrm{B}$ is the branching axiom which was first introduced in [10] and proved sound with respect to (divergence blind) branching congruence. The following lemma proves that B is also sound with respect to $={ }_{b}^{\Delta}$.

Lemma 3.1: Let $E, F \in \mathcal{E}, a \in A c t_{\tau}$. Then

$$
a \cdot(\tau \cdot(E+F)+F)=_{b}^{\Delta} a \cdot(E+F) .
$$

Proof. Directly follows from Lemma 2.12

$\mathrm{R} 0$ is the axiom of $\alpha$-conversion, which is known to be sound with respect to $\sim$, hence also sound with respect to $={ }_{b}^{\Delta}$ because of proposition 2.15 .

$\mathrm{R} 1-\mathrm{R} 3$ are three rules for recursion introduced in [1], and can be proved sound with respect to $\sim$, hence $\mathrm{R} 1$ and $\mathrm{R} 3$ are also sound with respect to $=\stackrel{\Delta}{b}$. As an equational rule with an equality as premise, the soundness of R2 with respect to $={ }_{b}^{\Delta}$ does not immediately follow from its soundness with respect to $\sim$, hence we need the following lemma for the soundness of $\mathrm{R} 2$ here.

Lemma 3.2: If $X$ is guarded in $E, F={ }_{b}^{\Delta} E\{F / X\}$, then $F={ }_{b}^{\Delta} \mu X . E$.

Proof. Construct the following relation:

$S=\{(H\{F / X\}, H\{\mu X . E / X\}) \mid H \in \mathcal{E}, X$ is guarded in $H\}$.

We show that $S \cup S^{-1}$ is a strong bisimulation up to $\approx_{b}^{\Delta}$. Once this is done, then since $(E\{F / X\}, E\{\mu X . E / X\}) \in S \cup S^{-1}$, it follows from Lemma 2.18 that $E\{F / X\}={ }_{b}^{\Delta} E\{\mu X . E / X\}$, and then $F={ }_{b}^{\Delta} E\{F / X\}={ }_{b}^{\Delta} E\{\mu X . E / X\}={ }_{b}^{\Delta} \mu X . E$.

To show that $S \cup S^{-1}$ is a strong bisimulation up to $\approx_{b}^{\Delta}$, suppose $(H\{F / X\}, H\{\mu X . E / X\}) \in S$ and $H\{F / X\} \stackrel{a}{\longrightarrow} L$. Then since $X$ is guarded in $H$, according to 1) of Lemma 2.2 it must be that $H \stackrel{a}{\longrightarrow} H^{\prime}$ and $L \equiv H^{\prime}\{F / X\}$, thus

$H\{\mu X . E / X\} \stackrel{a}{\longrightarrow} H^{\prime}\{\mu X . E / X\}$. If $a=\tau$, then $X$ must still be guarded in $H^{\prime}$, then $\left(H^{\prime}\{F / X\}, H^{\prime}\{\mu X . E / X\}\right) \in S$. If $a \neq \tau$, then $X$ could be unguarded in $H^{\prime}$. However, since $X$ is guarded in $E$, in this case $X$ is sill guarded in $H^{\prime}\{E / X\}$, and $H^{\prime}\{F / X\}={ }_{b}^{\Delta} H^{\prime}\{E\{F / X\} / X\}$, $\left(H^{\prime}\{E\{F / X\} / X\}, H^{\prime}\{E\{\mu X . E / X\} / X\}\right) \in S \cup S^{-1}$, $H^{\prime}\{E\{\mu X . E / X\} / X\}={ }_{b}^{\Delta} H^{\prime}\{\mu X . E / X\}$, thus

$\left(H^{\prime}\{F / X\}, H^{\prime}\{\mu X . E / X\}\right) \in \approx_{b}^{\Delta}\left(S \cup S^{-1}\right) \approx_{b}^{\Delta}$. Also since $X$ is guarded in $H$, it easily follows from 2) of Lemma 2.2 that if $H\{F / X\} \triangleright Y$ then $H\{\mu X . E / X\} \triangleright Y$.

If $(H\{F / X\}, H\{\mu X . E / X\}) \in S^{-1}$, in the same way we can show that the conditions 1),2),3) in Definition 2.17 are satisfied. So $S \cup S^{-1}$ is a strong bisimulation up to $\approx_{b}^{\Delta}$.

$\mathrm{R} 4$ as an axiom was first introduced in [11] for eliminating $\tau$ 's in front of unguarded occurrences of bound variables. For $={ }_{b}^{\Delta}$ however, R4 alone is not enough to eliminate all such $\tau$ 's. So here we introduce R5 to work together with R4. Intuitively, R5 means that, the presence of unguarded occurrences of $X$ in $E$ implies a $\tau$-circle going back to the recursion represented by $X$, thus the inner $\tau$-loop for the recursion represented by $Y$ can be eliminated without changing the divergent behaviour of the expression. The following lemma states the soundness of R4 and R5. The proof uses the technique of progressing branching bisimulation.

Lemma 3.3: Let $E, F, G \in \mathcal{E}, X, Y \in \mathcal{V}, E \tilde{\triangleright} X$, then

1) $\mu X .(\tau .(\tau . E+F)+G)={ }_{b}^{\Delta} \mu X .(\tau .(E+F)+G)$;

2) $\mu X .(\tau \cdot \mu Y .(\tau . Y+E)+F)={ }_{b}^{\Delta} \mu X .(\tau \cdot \mu Y . E+F)$.

Proof. To prove 1), let $L=\mu X .(\tau .(\tau . E+F)+G)$ and $R=\mu X .(\tau .(E+F)+G)$, and let $S$ be the following relation:

$$
\begin{aligned}
& \{(H\{L / X\}, H\{R / X\}) \mid H \in \mathcal{E}\} \cup \\
& \{(E\{L / X\}, E\{R / X\}+F\{R / X\}), \\
& (\tau . E\{L / X\}+F\{L / X\}, E\{R / X\}+F\{R / X\})\} .
\end{aligned}
$$

We show that $S \cup S^{-1}$ is a progressing branching bisimulation.

By Lemma 2.4 $L \stackrel{a}{\longrightarrow} L^{\prime}$ if and only if

1) either $G \stackrel{a}{\longrightarrow} G^{\prime}$ and $L^{\prime} \equiv G^{\prime}\{L / X\}$,

2) or $a=\tau$ and $L^{\prime} \equiv \tau . E\{L / X\}+F\{L / X\}$. 
For the same reason $R \stackrel{a}{\longrightarrow} R^{\prime}$ if and only if

1) either $G \stackrel{a}{\longrightarrow} G^{\prime}$ and $R^{\prime} \equiv G^{\prime}\{R / X\}$,

2) or $a=\tau$ and $R^{\prime} \equiv E\{R / X\}+F\{R / X\}$.

Because $E \tilde{\triangleright} X$, together with $L \stackrel{\tau}{\longrightarrow} \tau . E\{L / X\}+F\{L / X\}$ and $R \stackrel{\tau}{\longrightarrow} E\{R / X\}+F\{R / X\}$, the following follows from Lemma 2.3 .

1) $E\{L / X\} \stackrel{\tau}{\longrightarrow} \Longrightarrow \tau . E\{L / X\}+F\{L / X\}$, and

2) $E\{R / X\} \stackrel{\tau}{\longrightarrow} E E\{R / X\}+F\{R / X\}$.

From these observation, it is easy to check that $S \cup S^{-1}$ is a progressing branching bisimulation, hence a div.-pres. branching bisimulation by Lemma 2.9 , and $S \cup S^{-1} \subseteq \approx_{b}^{\Delta}$. Moreover, whenever $L \stackrel{a}{\longrightarrow} L^{\prime}$ then there exists $R^{\prime}$ such that $R \stackrel{a}{\longrightarrow} R^{\prime}$ with $\left(L^{\prime}, R^{\prime}\right) \in S$, and whenever $R \stackrel{a}{\longrightarrow} R^{\prime}$ then there exists $L^{\prime}$ such that $L \stackrel{a}{\longrightarrow} L^{\prime}$ with $\left(R^{\prime}, L^{\prime}\right) \in S^{-1}$, and $L \triangleright Y$ if and only if $R \triangleright Y$ for any $Y \in \mathcal{V}$, thus $L={ }_{b}^{\triangle} R$.

To prove 2), let $L_{X}=\mu X .(\tau . \mu Y .(\tau . Y+E)+F)$, $L_{Y}=\mu Y .\left(\tau . Y+E\left\{L_{X} / X\right\}\right), R_{X}=\mu X .(\tau . \mu Y . E+F)$, and $R_{Y}=\mu Y . E\left\{R_{X} / X\right\}$, and let $S$ be the following relation:

$$
\begin{aligned}
& \left\{\left(H\left\{L_{X} / X\right\}, H\left\{R_{X} / X\right\}\right) \mid H \in \mathcal{E}\right\} \cup \\
& \left\{\left(H\left\{L_{X} / X, L_{Y} / Y\right\}, H\left\{R_{X} / X, R_{Y} / Y\right\}\right) \mid H \in \mathcal{E}\right\} .
\end{aligned}
$$

We show that $S \cup S^{-1}$ is a progressing branching bisimulation.

By Lemma 2.4, $L_{X} \stackrel{a}{\longrightarrow} L^{\prime}$ if and only if

1) either $F \stackrel{a}{\longrightarrow} F^{\prime}$ and $L^{\prime} \equiv F^{\prime}\left\{L_{X} / X\right\}$,

2) or $a=\tau$ and $L^{\prime}$ is just $L_{Y}$.

And $L_{Y} \stackrel{a}{\longrightarrow} L^{\prime}$ if and only if

1) either $a=\tau$ and $L^{\prime}$ is just $L_{Y}$,

2) or $E \stackrel{a}{\longrightarrow} E^{\prime}$ and $L^{\prime} \equiv E^{\prime}\left\{L_{X} / X, L_{Y} / Y\right\}$,

3) or $L_{X} \stackrel{a}{\longrightarrow} L^{\prime}$, in which case either $F \stackrel{a}{\longrightarrow} F^{\prime}$ and $L^{\prime} \equiv F^{\prime}\left\{L_{X} / X\right\}$ or $a=\tau$ and $L^{\prime}$ is $L_{Y}$.

For the same reason $R \stackrel{a}{\longrightarrow} R^{\prime}$ if and only if

1) either $F \stackrel{a}{\longrightarrow} F^{\prime}$ and $R^{\prime} \equiv F^{\prime}\left\{R_{X} / X\right\}$,

2) or $a=\tau$ and $R^{\prime}$ is just $R_{Y}$.

And $R_{Y} \stackrel{a}{\longrightarrow} R^{\prime}$ if and only if

1) either $E \stackrel{a}{\longrightarrow} E^{\prime}$ and $R^{\prime} \equiv E^{\prime}\left\{L_{X} / X, L_{Y} / Y\right\}$,

2) or $R_{X} \stackrel{a}{\longrightarrow} R^{\prime}$, in which case either $F \stackrel{a}{\longrightarrow} F^{\prime}$ and $R^{\prime} \equiv F^{\prime}\left\{R_{X} / X\right\}$ or $a=\tau$ and $R^{\prime}$ is $R_{Y}$.

Because $E \tilde{\triangleright} X$, together with $R_{X} \stackrel{\tau}{\longrightarrow} R_{Y}$, it follows from Lemma 2.3 that $E\left\{R_{X} / X, R_{Y} / Y\right\} \stackrel{\tau}{\longrightarrow} \Longrightarrow R_{Y}$, thus $R_{Y} \stackrel{\tau}{\longrightarrow} \Longrightarrow R_{Y}$. From these observation, it is easy to check that $S \cup S^{-1}$ is a progressing branching bisimulation, hence a div.-pres. branching bisimulation, and $S \cup S^{-1} \subseteq \approx_{b}^{\Delta}$. Moreover, whenever $L_{X} \stackrel{a}{\longrightarrow} L^{\prime}$ then there exists $R^{\prime}$ such that $R_{X} \stackrel{a}{\longrightarrow} R^{\prime}$ with $\left(L^{\prime}, R^{\prime}\right) \in S$, and whenever $R_{X} \stackrel{a}{\longrightarrow} R^{\prime}$ then there exists $L^{\prime}$ such that $L_{X} \stackrel{a}{\longrightarrow} L^{\prime}$ with $\left(R^{\prime}, L^{\prime}\right) \in S^{-1}$, and $L_{X} \triangleright Z$ if and only if $R_{X} \triangleright Z$ for any $Z \in \mathcal{V}$, thus $L_{X}={ }_{b}^{\Delta} R_{X}$.

A key idea proposed by Milner in [2] for equational axiomatisation of this kind is to transform arbitrary expressions into guarded ones, i.e. expressions in which every recursive subexpression is a guarded recursion, so that the fixed point induction rule $\mathrm{R} 2$ can be applied to derive equality of semantically equivalent expressions. Determined by its divergence preserving nature, a major difficulty in an axiomatisation for $={ }_{b}^{\Delta}$ is that unguarded recursions cannot be eliminated completely like in the axiomatisation for $=_{b}$. Thus, in order to use the full power of the fixed point induction rule $\mathrm{R} 2$, careful manipulation of unguarded recursions is called for, and axioms $\mathrm{R} 6, \mathrm{R} 7$, and $\mathrm{R} 8$ are exactly for that purpose. The intuition for $\mathrm{R} 6$ is that, the left hand side expression is a recursion starting with a $\tau$, while the right hand side expression always perform a $\tau$ before recursion, so they should be doing the same thing. $\mathrm{R} 7$ roughly says that double loop is the same as a single loop, which intuitively makes sense. The intuition for $\mathrm{R} 8$ is that here the successive recursion for $X$ and $Y$ effectively defines $X$ and $Y$ as the same behaviour, thus interchanging the two variables should not affect the overall behaviour. The following lemma states the soundness of $\mathrm{B} 6, \mathrm{R} 7$, and $\mathrm{R} 8$ with respect to $={ }_{b}^{\Delta}$. Surprisingly it turned out that these axioms are even sound with respect to the stronger congruence $\sim$.

Lemma 3.4: Let $E, F \in \mathcal{E}, X, Y \in \mathcal{V}$. Then

1) $\mu X . \tau . E \sim \tau . \mu X . E\{\tau . X / X\}$;

2) $\mu X .(\tau . X+\mu Y .(\tau . Y+E)) \sim \mu X . \mu Y(\tau . Y+E)$;

3) $\mu X . \mu Y .(\tau .(X+E)+F) \sim \mu X . \mu Y .(\tau .(Y+E)+F)$.

Proof. To prove 1), let

$S=\{(H\{\mu X . \tau . E / X\}, H\{\tau . \mu X . E\{\tau . X / X\} / X\} \mid H \in \mathcal{E}\}$.

Then $S \cup S^{-1}$ is a strong bisimulation up to $\sim$, thus $S \subseteq \sim$. Take $X$ as $H$, then $(\mu X . \tau . E, \tau . \mu X . E\{\tau . X / X\}) \in S$, thus $\mu X . \tau . E \sim \tau . \mu X . E\{\tau . X / X\}$.

To prove 2), let

$$
S=\left\{\left(H\left\{L_{X} / X, L_{Y} / Y\right\}, H\left\{R_{X} / X, R_{Y} / Y\right\}\right) \mid H \in \mathcal{E}\right\}
$$

where

$L_{X}=\mu X .(\tau . X+\mu Y .(\tau . Y+E)), L_{Y}=\mu Y .\left(\tau . Y+E\left\{L_{X} / X\right\}\right)$, $R_{X}=\mu X . \mu Y .(\tau . Y+E), \quad R_{Y}=\mu Y .\left(\tau . Y+E\left\{R_{X} / X\right\}\right)$. Note that $R_{X} \sim R_{Y}$, then $S \cup S^{-1}$ is a strong bisimulation up-to $\sim$, thus $S \subseteq \sim$ follows from Lemma 2.18 Take $X$ as $H$, we have $\left(L_{X}, R_{X}\right) \in S$, hence $\mu X .(\tau . X+\mu Y .(\tau . Y+E)) \sim \mu X . \mu Y(\tau . Y+E)$.

To prove 3$)$, let

$$
S=\left\{\left(H\left\{L_{X} / X, L_{Y} / Y\right\}, H\left\{R_{X} / X, R_{Y} / Y\right\}\right) \mid H \in \mathcal{E}\right\}
$$

where

$L_{X}=\mu X \cdot \mu Y .(\tau \cdot(X+E)+F)$,

$L_{Y}=\mu Y .\left(\tau .\left(L_{X}+E\left\{L_{X} / X\right\}\right)+F\left\{L_{X} / X\right\}\right)$,

$R_{X}=\mu X . \mu Y .(\tau .(Y+E)+F)$,

$R_{Y}=\mu Y .\left(\tau .\left(Y+E\left\{R_{X} / X\right\}\right)+F\left\{R_{X} / X\right\}\right)$.

Note that $R_{X} \sim R_{Y}$, then $S \cup S^{-1}$ is a strong bisimulation up-to $\sim$, thus $S \subseteq \sim$. Take $X$ as $H$ to obtain $\left(L_{X}, R_{X}\right) \in S$, hence $\mu X . \mu Y .(\tau .(X+E)+F) \sim \mu X . \mu Y .(\tau .(Y+E)+F)$.

With these lemmas, finally we have the following soundness theorem for the inference system with respect to $={ }_{b}^{\Delta}$.

Theorem 3.5: For $E, F \in \mathcal{E}$, if $\vdash E=F$ then $E={ }_{b}^{\Delta} F$.

Proof. Since $=\Delta b$ is an equivalence relation, equational reasoning preserves soundness. Also by Theorem $2.16={ }_{b}^{\Delta}$ is a congruence, thus the inference rule of substituting equal for 
equal preserves soundness. We also know the soundness of S1-S4, B, R0-R7. Thus if $\vdash E=F$ then $E={ }_{b}^{\Delta} F$.

Before closing this section we prove two useful derived rules. Milner's inference system in [2] included the following

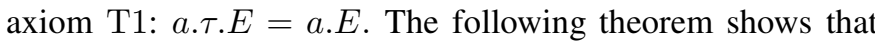
with $\mathrm{B}, \mathrm{T} 1$ can be derived from the present axiomatisation.

Theorem 3.6: Let $E \in \mathcal{E}$. Then:

$$
\mathrm{T} 1 \vdash \text { a.t.E }=\text { a.E. }
$$

Proof. $\vdash a . \tau . E=a .(\tau .(E+\mathbf{0})+\mathbf{0})=a \cdot(E+\mathbf{0})=a . E$.

Thus $\mathrm{T} 1$ can be used as a derived rule in the inference system.

The next derived rule is D0 as stated in Theorem 3.8. To prove it we need the following lemma.

Lemma 3.7: Let $E, E^{\prime} \in \mathcal{E}, X \in \mathcal{V}$.

1) If $E \stackrel{a}{\longrightarrow} E^{\prime}$ then $\vdash E=E+a . E^{\prime}$;

2) If $E \triangleright X$ then $\vdash E=E+X$.

Proof. By straightforward induction on the set of rules defining the relations $\longrightarrow$ and $\triangleright$ (Definition 2.1).

Theorem 3.8: For $E, F \in \mathcal{E}, X \in \mathcal{V}$, the following holds: D0 If $E \tilde{\triangleright} X$ then $\vdash \mu X .(\tau . E+F)=\mu X .(\tau .(X+E)+F)$. Proof. Since $E \tilde{\triangleright} X$ is $E \Longrightarrow \triangleright X$, i.e. there exist $E_{1}, \ldots, E_{n}$ such that $E_{i-1} \stackrel{\tau}{\longrightarrow} E_{i}$ for $i=1, \ldots, n\left(E_{0} \equiv E\right)$ and $E_{n} \triangleright X$. By Lemma 3.7 $\vdash E_{i-1}=E_{i-1}+\tau$. $E_{i}$ for $i=1, \ldots, n$ and $\vdash E_{n}=E_{n}+X$. Then we have the following proof:

$$
\vdash \mu X .(\tau . E+F)=\mu X .\left(\tau . E_{0}+F\right)
$$$$
=\mu X \cdot\left(\tau \cdot\left(E_{0}+\tau \cdot E_{1}\right)+F\right)
$$$$
=\mu X .\left(\tau \cdot\left(E_{0}+E_{1}\right)+F\right)
$$$$
=\mu X \cdot\left(\tau \cdot\left(E_{0}+\ldots+E_{n}\right)+F\right)
$$$$
=\mu X .\left(\tau .\left(E_{0}+\ldots+E_{n}+X\right)+F\right)
$$
repeating above step

$=\mu X \cdot\left(\tau \cdot\left(X+E_{0}+\ldots+E_{n-1}+E_{n}\right)+F\right)$ Lemma 3.7

$=\mu X .\left(\tau .\left(X+E_{0}+\ldots+E_{n-1}+\tau \cdot E_{n}\right)+F\right)$

$=\mu X \cdot\left(\tau \cdot\left(X+E_{0}+\ldots+E_{n-1}\right)+F\right)$

$=\mu X \cdot\left(\tau \cdot\left(X+E_{0}\right)+F\right)$

$=\mu X \cdot(\tau \cdot(X+E)+F)$

\section{LOOP OPERATOR AND STANDARD SUM}

The loop operator $\tau^{*}$ applied on an expression $E$ obtains the loop expression $\tau^{*} E$ which can choose to perform $\tau$ without changing its state or choose to perform actions of $E$. In [13], the loop expression $\tau^{*} E$ was included into the basic syntax of expressions (where the notation used is $\Delta(E)$ ), and played a key role in defining (guarded) standard forms for all expressions. The syntax of basic expressions of this paper does not include the loop operator $\tau^{*}$, we introduce the following definition instead.

Definition 4.1: Let $E$ be an expression, define the loop expression $\tau^{*} E$ as $\mu X$. $(\tau X+E)$ where $X \notin F V(E)$.

That is we use $\tau^{*} E$ as an abbreviation, then we can reuse the generalized notion of guarded expression introduced in [13]. Excluding the loop operator from the basic syntax helps to simplify the semantic theory (so there is no need to define particularly the operational semantics of $\tau^{*} E$, and to study the congruence property concerning $\tau^{*} E$ etc.) and to keep a small set of core axioms of the inference system.

In this section we first prove some derived rules relating to the loop operator. These derived rules, together with T1
(Theorem 3.6) and D0 (Theorem [3.8), will be used in the standardization process.

Theorem 4.2: The following equalities can be derived from the inference system.

$\mathrm{D} 1 \vdash \tau^{*} E=\tau .\left(\tau^{*} E\right)+E$;

$\mathrm{D} 2 \vdash \tau^{*} E=\tau^{*} E+E$;

D3 $\vdash \mu X .(\tau .(X+E)+F)=\mu X .\left(\tau . \tau^{*}(E+F)+F\right)$;

$\mathrm{D} 4 \vdash \mu X .(\tau .(X+E)+\tau .(X+F)+G)=\mu X .(\tau \cdot(X+E+F)+G)$;

D5 $\vdash \tau^{*}\left(\tau \cdot \tau^{*}(E+F)+F\right)=\tau . \tau^{*}(E+F)+F$;

D6 $\vdash \tau^{*}\left(\tau^{*} E\right)=\tau^{*} E$.

Proof. D1 is a special instance of R1.

The following is a proof of D2:

$\begin{array}{lr}\vdash \tau^{*} E=\tau \cdot \tau^{*} E+E & \text { D1 } \\ =\tau \cdot \tau^{*} E+E+E & \text { S3 } \\ =\tau^{*} E+E & \text { D1 }\end{array}$

The following is a proof of D3, where $Y$ is a variable which occurs neither free in $E$ nor in $F$ :

$\vdash \mu X .(\tau .(X+E)+F)$

$=\mu X \cdot \mu Y \cdot(\tau \cdot(X+E)+F)$

$\mathrm{R} 1$

$=\mu X \cdot \mu Y \cdot(\tau \cdot(Y+E)+F)$

$\mathrm{R} 8$

$=\mu X \cdot \mu Y \cdot(\tau \cdot(\tau \cdot Y+E)+F)$

$\mathrm{R} 4$

$=\mu X \cdot(\tau \cdot(\tau \cdot \mu Y \cdot(\tau \cdot(\tau . Y+E)+F)+E)+F)$

$\mathrm{R} 1$

$=\mu X .(\tau \cdot(\mu Y \cdot \tau \cdot(\tau \cdot(Y+E)+F)+E)+F) \quad$ R6

$=\mu X .(\tau .(\mu Y . \tau .(Y+E+F)+E)+F) \quad$ R4

$=\mu X \cdot(\tau \cdot(\tau \cdot \mu Y \cdot(\tau . Y+E+F)+E)+F)$

$\mathrm{R} 6$

$=\mu X \cdot\left(\tau \cdot\left(\tau \cdot \tau^{*}(E+F)+E\right)+F\right)$

Definition 4.1

$=\mu X \cdot\left(\tau \cdot\left(\tau \cdot\left(\tau^{*}(E+F)+E+F\right)+E\right)+F\right) \quad$ D2

$=\mu X \cdot\left(\tau \cdot\left(\tau^{*}(E+F)+E+F\right)+F\right)$

$=\mu X \cdot\left(\tau \cdot \tau^{*}(E+F)+F\right)$

D2

The following is a proof of $\mathrm{D} 4$, where $Y$ is a variable which does not occur free in $E, F, G$ :

$\vdash \mu X .(\tau .(X+E)+\tau .(X+F)+G)$

$=\mu X .\left(\tau \cdot \tau^{*}(E+\tau \cdot(X+F)+G)+\tau \cdot(X+F)+G\right)$

$=\mu X \cdot(\tau \cdot \mu Y \cdot(\tau \cdot Y+E+\tau \cdot(X+F)+G)+\tau \cdot(X+F)+G)$

$=\mu X \cdot(\tau \cdot \mu Y \cdot(E+\tau \cdot(X+F)+G)+\tau \cdot(X+F)+G) \quad \mathrm{R} 5$

$=\mu X .(\tau \cdot(E+\tau \cdot(X+F)+G)+\tau \cdot(X+F)+G)$

$=\mu X \cdot(\tau \cdot(E+(X+F)+G)+\tau \cdot(X+F)+G)$

$\mathrm{R} 4, \mathrm{~S} 1$

$=\mu X .(\tau \cdot(X+E+F+G)+\tau \cdot(X+F)+G)$

$\mathrm{S} 1, \mathrm{~S} 2$

$=\mu X .(\tau \cdot(X+F)+\tau \cdot(X+E+F+G)+G)$

$=\mu X \cdot\left(\tau \cdot \tau^{*}(F+\tau \cdot(X+E+F+G)+G)\right.$

$$
+\tau \cdot(X+E+F+G)+G)
$$

$=\mu X \cdot(\tau \cdot(F+\tau \cdot(X+E+F+G)+G)$

$$
+\tau \cdot(X+E+F+G)+G)
$$

$=\mu X \cdot(\tau \cdot(F+(X+E+F+G)+G)$ $+\tau \cdot(X+E+F+G)+G)$

$\mathrm{R} 4, \mathrm{~S} 1$

$=\mu X .(\tau \cdot(X+E+F+G)+\tau .(X+E+F+G)+G)$ S1-S3

$=\mu X \cdot(\tau \cdot(X+E+F+G)+G)$

$=\mu X \cdot\left(\tau \cdot \tau^{*}(E+F+G)+G\right)$

$=\mu X \cdot(\tau \cdot(X+E+F)+G)$

The following is a proof of D5, where $X, Y$ are different variables which occur neither free in $E$ nor in $F$ :

$\vdash \tau^{*}\left(\tau \cdot \tau^{*}(E+F)+F\right)$

$=\mu X \cdot\left(\tau \cdot X+\tau \cdot \tau^{*}(E+F)+F\right)$

$=\mu X \cdot\left(\tau \cdot X+\mu Y \cdot\left(\tau \cdot \tau^{*}(E+F)+F\right)\right)$

Definition 4.1 


$$
\begin{aligned}
& =\mu X \cdot(\tau \cdot X+\mu Y \cdot(\tau \cdot(Y+E)+F)) \\
& =\mu X \cdot(\tau \cdot X+\mu Y \cdot(\tau \cdot Y+\tau \cdot(Y+E)+F)) \\
& =\mu X \cdot \mu Y \cdot(\tau \cdot Y+\tau \cdot(Y+E)+F) \\
& =\mu X \cdot \mu Y \cdot(\tau \cdot(Y+E)+F) \\
& =\mu X \cdot \mu Y \cdot\left(\tau \cdot \tau^{*}(E+F)+F\right) \\
& =\mu X \cdot\left(\tau \cdot \tau^{*}(E+F)+F\right) \\
& =\tau \cdot \tau^{*}(E+F)+E
\end{aligned}
$$

D6 can be derived from D1 and D5 as follows:

$\vdash \tau^{*}\left(\tau^{*} E\right)=\tau^{*}\left(\tau . \tau^{*} E+E\right)$

$=\tau^{*}\left(\tau \cdot \tau^{*}(\mathbf{0}+E)+E\right)$

$\left.=\tau \cdot \tau^{*}(\mathbf{0}+E)+E\right)$

$=\tau \cdot \tau^{*} E+E=\tau^{*} E$

$\mathrm{D} 4, \mathrm{~S} 3$

Definition 4.3: A guarded recursion is an expression of the form $\mu X$. $E$ where $X$ is guarded in $E$.

A guarded expression is an expression in which every subexpression of the form $\mu X . E$ is either a loop expression or a guarded recursion.

Let $E \in \mathcal{E}, X \in \mathcal{V}, X$ is said fully exposed in $E$ if whenever an unguarded occurrence of $X$ in $E$ is within a subexpression $\mu Y . F$ of $E$, then $\mu Y . F$ is a loop expression.

The main purpose of this section is to prove the following standardization result: every expression can be proven equivalent to a guarded expression of some form. In the standardization process, the property that whether a variable $X$ is fully exposed in an expression $E$ is very important. If $X$ is not fully exposed in a guarded expression, then there is an equivalent guarded expression in which $X$ is fully exposed. For example, the occurrence $X$ in $E \equiv \tau . \mu Y .(\tau . X+a . Y)$ is unguarded, and since it is within a recursion which is not a loop expression, $X$ is not fully exposed in $E$. Applying R1 to unfold the recursion, we obtain $\vdash E=\tau$. $(\tau . X+a . \mu Y .(\tau . X+a . Y))$, and $X$ becomes fully exposed on the right hand side (suppose $a \neq \tau$ ). The following lemma shows that this is generally true.

Lemma 4.4: Let $E \in \mathcal{E}, X \in \mathcal{V}$. If $E$ is a guarded expression, then there exists a guarded expression $E^{\prime}$ such that $X$ is fully exposed in $E^{\prime}$ and $\vdash E=E^{\prime}$.

Proof. The proof is by induction on the structure of $E$. Here we look at the only non trivial case where $E$ is a recursion $\mu Y . F$. Since $\mu Y . F$ is a guarded expression, either it is a loop expression so $F \equiv \tau . Y+F_{1}$ where $F_{1}$ is a guarded expression, or $Y$ is guarded in $F$ which itself is a guarded expression. In the first case, by the induction hypothesis there exists a guarded expression $F_{1}^{\prime}$ such that $X$ is fully exposed in $F_{1}^{\prime}$ and $\vdash F_{1}=F_{1}^{\prime}$. Then $\mu Y .\left(\tau . Y+F_{1}^{\prime}\right)$ (which is a loop expression) is guarded and $X$ is fully exposed in $\mu Y .\left(\tau . Y+F_{1}^{\prime}\right)$ and $\vdash \mu Y . F=\mu Y .\left(\tau . Y+F_{1}^{\prime}\right)$. In the second case, by the induction hypothesis there exists a guarded expression $F^{\prime}$ such that $X$ is fully exposed in $F^{\prime}$ and $\vdash F=F^{\prime}$. Since $Y$ is guarded in $F$ and $F={ }_{b}^{\Delta} F^{\prime}$ (follows from $\vdash F=F^{\prime}$ ), $Y$ must be guarded also in $F^{\prime}$. Then $X$ is fully exposed in $F^{\prime}$ implies that $X$ is fully exposed in $F^{\prime}\left\{\mu Y . F^{\prime} / Y\right\}$ since those unexposed unguarded occurrences of $X$ in $\mu Y . F^{\prime}$ becomes guarded occurrences in $F^{\prime}\left\{\mu Y . F^{\prime} / Y\right\}$. Clearly $F^{\prime}\left\{\mu Y . F^{\prime} / Y\right\}$ is guarded since $F^{\prime}$ is guarded. Now $\vdash \mu Y . F^{\prime}=F^{\prime}\left\{\mu Y . F^{\prime} / Y\right\}$, thus $\vdash \mu Y . F=F^{\prime}\left\{\mu Y . F^{\prime} / Y\right\}$ and $F^{\prime}\left\{\mu Y . F^{\prime} / Y\right\}$ is the $E^{\prime}$ we need in this case.
Lemma 4.5: Let $E, F \in \mathcal{E}, X \in \mathcal{V}$. If $E$ is guarded, $E \tilde{\triangleright} X$, and $X$ is fully exposed in $E$, then there exists $E_{1}$ such that $X$ is guarded in $E_{1}$, and $\vdash \mu X .(\tau . E+F)=\mu X .\left(\tau .\left(X+E_{1}\right)+F\right)$.

Proof. The proof is by induction on the structure of $E$.

If $E$ is $\mathbf{0}$, then $E \not \triangleright X$, and in this case the claim holds vacuously.

If $E$ is a variable, then since $E \tilde{\nabla} X$ the variable must be $X$, we can take 0 as $E_{1}$, then $X$ is guarded in $E_{1}$ and the equality holds and $E_{1}$ is a guarded expression.

If $E$ is a prefix form $a \cdot E^{\prime}$, since $E \tilde{\triangleright} X$, it must be that $a=\tau$ and $E^{\prime} \tilde{\nabla} X$. Since $X$ is fully exposed in $E, X$ must be fully exposed in $E^{\prime}$. Then

$$
\begin{aligned}
& \vdash \mu X .(\tau \cdot E+F)=\mu X \cdot\left(\tau \cdot \tau \cdot E^{\prime}+F\right) \\
& =\mu X .\left(\tau \cdot E^{\prime}+F\right) \\
& =\mu X .\left(\tau \cdot\left(X+E_{1}\right)+F\right)
\end{aligned}
$$

ind. hyp. on $E^{\prime}$

where $E_{1}$ is obtained by applying induction hypothesis on $E^{\prime}$, such that $E_{1}$ is a guarded expression, and $X$ is guarded in $E_{1}$.

If $E$ is $E^{\prime}+E^{\prime \prime}$, then $E \tilde{\triangleright} X$ and $X$ is fully exposed in $E$ implies that $X$ is fully exposed in $E^{\prime}$ and $E^{\prime \prime}$, and either $E^{\prime} \tilde{\triangleright} X$ or $E^{\prime \prime} \tilde{\triangleright} X$ or both. If $E^{\prime} \tilde{\triangleright} X$ and $X$ is fully exposed in $E^{\prime}$, then

$$
\begin{aligned}
& \vdash \mu X .(\tau . E+F)=\mu X .(\tau \cdot(X+E)+F) \\
& =\mu X .\left(\tau \cdot\left(X+E^{\prime}+E^{\prime \prime}\right)+F\right) \\
& =\mu X \cdot\left(\tau \cdot\left(X+E^{\prime}\right)+\tau\left(X+E^{\prime \prime}\right)+F\right) \\
& =\mu X \cdot\left(\tau \cdot E^{\prime}+\tau \cdot\left(X+E^{\prime \prime}\right)+F\right) \\
& =\mu X .\left(\tau \cdot\left(X+E_{1}^{\prime}\right)+\tau \cdot\left(X+E^{\prime \prime}\right)+F\right) \text { ind. hyp. on } E^{\prime} \\
& =\mu X \cdot\left(\tau \cdot\left(X+E_{1}^{\prime}+E^{\prime \prime}\right)+F\right)
\end{aligned}
$$

where $E_{1}^{\prime}$ is a guarded expression obtained by applying induction hypothesis on $E^{\prime}$, and $X$ is guarded in $E_{1}^{\prime}$. If $X$ is guarded in $E^{\prime \prime}$ then $E_{1}^{\prime}+E^{\prime \prime}$ is the final $E_{1}$ which is a guarded expression and in which $X$ is guarded, otherwise we can do the same routine again on $E^{\prime \prime}$.

If $E$ is a recursion $\mu Y . H$, since $E \tilde{\triangleright} X$ and $X$ is fully exposed in $E, \mu Y . H$ must be a loop expression, that is $\mu Y . H \equiv \tau^{*} E^{\prime}$, and $E^{\prime} \tilde{\nabla} X$, and $X$ is fully exposed in $E^{\prime}$. Then:

$$
\begin{aligned}
& \vdash \mu X \cdot(\tau \cdot E+F)=\mu X \cdot\left(\tau \cdot \tau^{*} E^{\prime}+F\right) \\
& =\mu X \cdot\left(\tau \cdot E^{\prime}+F\right) \\
& =\mu X \cdot\left(\tau \cdot\left(X+E_{1}^{\prime}\right)+F\right)
\end{aligned}
$$
ind. hyp. on $E^{\prime}$ where $E_{1}^{\prime}$ is a guarded expression obtained by applying induction hypothesis on $E^{\prime}$, and $X$ is guarded in $E_{1}^{\prime}$.

Let $S=\left\{E_{1}, \ldots, E_{n}\right\}$ be a finite set of expressions, we write $\Sigma S$ and $\Sigma_{i=1}^{n} E_{i}$ as abbreviations for $E_{1}+\ldots+E_{n}$. The use of such notations is justified by the axioms S1-S4.

Definition 4.6: A standard sum is an expression of the form $\sum_{i=1}^{n} a_{i} \cdot E_{i}+\sum_{j=1}^{m} W_{j}$ where $E_{i}$ is a guarded expression for $i=1, \ldots, n$ and $W_{j} \in \mathcal{V}$ for $j=1, \ldots, m$.

A simple standard sum (or a simple sum) is a standard sum of the form $\Sigma_{i=1}^{n} a_{i} . X_{i}+\sum_{j=1}^{m} W_{j}$ where $X_{i}$ 's and $W_{j}$ 's are all variables.

At last we can state and prove the standardization theorem.

Theorem 4.7: Every expression can be proven equivalent to a standard sum.

Proof. We prove by structural induction that for every $E \in \mathcal{E}$ there is a standard sum $E^{\prime}$ such that $\vdash E=E^{\prime}$. 
Here we only look at the case where $E$ is a recursion $\mu X . F$, all other cases are simple. By the induction hypothesis there exist guarded expressions $E_{1}, \ldots, E_{n}$ and variables $W_{1}, \ldots, W_{m}$ such that $\vdash F=\sum_{i=1}^{n} a_{i} . E_{i}+\sum_{j=1}^{m} W_{j}$. Let $S$ be the set of summands in $\sum_{i=1}^{n} a_{i} . E_{i}+\sum_{j=1}^{m} W_{j}$, then the elements of $S$ can be divided into four groups:

1) those of the form $a . H$ where $X$ occurs unguarded in a.H;

2) those of the form $a . H$ where $X$ is guarded in $a . H$;

3) those variable $W$ which is not $X$;

4) $X$ (if it is one of the summands).

Let $G$ be the sum of all expressions in the second and third groups above, then $G$ is a standard guarded sum and $X$ is guarded in $G$, and let $\tau . F_{1}, \ldots, \tau . F_{k}$ be the expressions in the first group (since $X$ occurs unguarded in these summands, the prefix must be $\tau$ ), then $\vdash \mu X . F=\mu X .\left(\sum_{i=1}^{k} \tau . F_{i}+G\right)$ (using R3 to eliminate $X$ in the fourth group if needed). Since each $F_{i}$ is a guarded expression, by Lemma 4.4 there is a guarded expression $F_{i}^{\prime}$ s. t. $X$ is fully exposed in $F_{i}^{\prime}$ and $\vdash F_{i}=F_{i}^{\prime}$, thus $\vdash \mu X . F=\mu X .\left(\sum_{i=1}^{k} \tau . F_{i}^{\prime}+G\right)$. Also note that since the $\tau . F_{i}$ 's are from the first group, thus $X$ occurs unguarded in $F_{i}$, so $F_{i} \tilde{\triangleright} X$ (Lemma2.3). Then $F_{i}^{\prime} \tilde{\triangleright} X$ follows from Lemma 2.13 Now apply Lemma $4.5 k$ times we find guarded expressions $F_{1}^{\prime \prime}, \ldots, F_{k}^{\prime \prime}$ such that $X$ is guarded in all $F_{i}^{\prime \prime}$ 's and

$$
\begin{aligned}
& \vdash \mu X .\left(\sum_{i=1}^{k} \tau . F_{i}^{\prime}+G\right)=\mu X .\left(\sum_{i=1}^{k} \tau .\left(X+F_{i}^{\prime \prime}\right)+G\right) \text {. Then } \\
& \vdash \mu X . F=\mu X .\left(\sum_{i=1}^{k} \tau .\left(X+F_{i}^{\prime \prime}\right)+G\right) \\
& =\mu X \cdot\left(\tau \cdot\left(X+\sum_{i=1}^{k} F_{i}^{\prime \prime}\right)+G\right) \\
& =\mu X \cdot\left(\tau \cdot \tau^{*}\left(\sum_{i=1}^{k} F_{i}^{\prime \prime}+G\right)+G\right) \\
& =\tau \cdot \tau^{*}\left(\sum_{i=1}^{k} F_{i}^{\prime \prime}\{L / X\}+G\{L / X\}\right)+G\{L / X\}
\end{aligned}
$$

where $L \equiv \mu X .\left(\tau \cdot \tau^{*}\left(\sum_{i=1}^{k} F_{i}^{\prime \prime}+G\right)+G\right)$. Now $L$ is a guarded expression since $G$ and $F_{1}^{\prime \prime}, \ldots, F_{k}^{\prime \prime}$ are guarded expressions and $X$ is guarded in $G$ and $F_{1}^{\prime \prime}, \ldots, F_{k}^{\prime \prime}$. Moreover since $G$ is a standard guarded sum, so are $G\{L / X\}$ and $\tau . \tau^{*}\left(\sum_{i=1}^{k} F_{i}^{\prime \prime}\{L / X\}+G\{L / X\}\right)+G\{L / X\}$.

\section{Quotient of Standard Equation System}

Definition 5.1: A recursive equation system $S$ is a finite set of equations

$$
\left\{X_{i}=F_{i} \mid i=1, \ldots, n\right\}
$$

where $X_{1}, \ldots, X_{n} \in \mathcal{V}$ are $n$ different variables, called the formal variables of $S$, and $F_{i} \in \mathcal{E}$ for $i=1, \ldots, n$.

For $E \in \mathcal{E}, E$ is said to provably solve (or satisfy) the recursive equation system $S$ above for variable $X_{k}$ with $1 \leq k \leq n$ if there are expressions $E_{i}$ for $i=1, \ldots, n$ with $E$ being $E_{k}$, such that $\vdash E_{i}=F_{i}\left\{E_{1} / X_{1}, \ldots, E_{n} / X_{n}\right\}$ for $i=1, \ldots, n$.

Let $X, Y$ be two formal variables of a recursive equation system $S, Y$ is said $S$-unguarded for $X$, written $X \tilde{\triangleright}_{S} Y$, if $Y$ occurs unguarded in the defining expression of $X$ in $S$, i.e. $X=F_{X} \in S$ and $F_{X} \tilde{\triangleright} Y$. A recursive equation system is said guarded if $\tilde{\triangleright}_{S}$ is a well-founded relation between the formal variables of $S$.

Theorem 5.2: Let $S$ be a recursive equation system, then for every formal variable of $S$ there is a provable solution. Moreover, if $S$ is guarded, and both $D, E$ are provable solutions of
$S$ for the same formal variable $X$, then $\vdash E=D$, i.e. every guarded recursive equation system has unique solution up to provability.

Proof. See [2], where only axioms valid in our inference system were used.

A particular kind of recursive equation system is standard equation system.

Definition 5.3: A standard equation system (or standard equation set), noted SES, is a guarded recursive equation system in which the right hand side of each equation has either of the following forms

$$
\begin{aligned}
& \text { (1) } \sum_{i=1}^{n} a_{i} X_{i}+\sum_{j=1}^{m} W_{j} \\
& \text { (2) } \tau^{*}\left(\sum_{i=1}^{n} a_{i} \cdot X_{i}+\sum_{j=1}^{m} W_{j}\right)
\end{aligned}
$$

where the $X_{i}$ 's are formal variables of $S$, and $W_{j}$ 's are not formal variables of $S$.

Definition 5.4: Let $X, X^{\prime} \in \mathcal{V}$ be two formal variables of a guarded recursive equation system $S$.

$X$ and $X^{\prime}$ are said to have equivalent solutions of $S$ if whenever $E, E^{\prime} \in \mathcal{E}$ are provable solutions of $S$ for $X$ and $X^{\prime}$ respectively then $E \approx_{b}^{\Delta} E^{\prime}$, and in this case we write $X \approx_{S} X^{\prime}$.

$S$ is said to have common provable solution for $X$ and $X^{\prime}$ if there exists $E \in \mathcal{E}$ which provable solves $S$ for $X$ as well as for $X^{\prime}$.

A formal variable $X$ of $S$ is called a bottom variable if whenever $X \tilde{\triangleright}_{S} Y$ for some formal variable $Y$ of $S$ then $X \varkappa_{S} Y$.

The following lemma shows that when $S$ is guarded, then $\approx_{S}$ is (as the symbol suggests) an equivalence relation between the formal variables of $S$.

Lemma 5.5: Let $S$ be a guarded recursive equation system.

$1) \approx_{S}$ is an equivalence relation between the formal variables of $S$;

2) for any formal variable $X$ of $S,[X]$ contains a bottom variable, where $[X]$ is the $\approx_{S}$-equivalence class containing $X$.

Proof. We first prove 1). Since $S$ is guarded, Theorem 5.2 guarantees unique solution for every formal variable $X$, which implies reflexivity of $\approx_{S}$. Symmetry is obvious by the symmetric phrase in the definition. Transitivity easily follows from the transitivity of $\approx_{b}^{\Delta}$.

We now turn to 2). By the guardedness of $S$ we know that $\tilde{\nabla}_{S}$ is a well-founded relation between formal variables of $S$. We prove the lemma by well-founded induction on $\tilde{\triangleright}_{S}$. If $X$ is a bottom element, then of course $[X]$ contains $X$ which is a bottom element. If $X$ is not a bottom element, then there is a formal variable $X^{\prime}$ such that $X^{\prime} \in[X]$ and $X \tilde{\triangleright}_{S} X^{\prime}$. By the induction hypothesis $\left[X^{\prime}\right]$ contains a bottom element, thus $[X]$ contains a bottom element since $[X]=\left[X^{\prime}\right]$.

The notion of bottom variable resembles the notion of bottom elements of branching bisimulation equivalence class introduced in [7].

The main purpose of this section is to use the quotient construction to prove Theorem 5.16, which states that the $\approx_{S}$-equality between formal variables of a standard equation system $S$ implies common solution for the formal variables of 
a related equation system. We need some preparation to prove the theorem. The next three lemmas state some important properties of bottom variables of SES.

Lemma 5.6: Let $S$ be an SES, $X=F_{X} \in S$. If $X$ is a bottom variable and $F_{X} \stackrel{\tau}{\longrightarrow} X^{\prime}$, then $X^{\prime} \not_{S} X$.

Proof. In this case $X \tilde{\triangleright}_{S} X^{\prime}$, and since $X$ is a bottom variable, $X^{\prime} \nsim_{S} X$.

Lemma 5.7: Let $S$ be an SES, $X, Y$ be two formal variables of $S$ with $X=F_{X}, Y=F_{Y} \in S$. If $X$ is a bottom variable and $X \approx_{S} Y$, then

1) whenever $F_{Y} \triangleright W$ then $F_{X} \triangleright W$;

2) whenever $F_{Y} \stackrel{a}{\longrightarrow} Y^{\prime}$ where either $Y^{\prime} \not \varpi_{S} X$ or $a \neq \tau$, then there exists $X^{\prime}$ such that $F_{X} \stackrel{a}{\longrightarrow} X^{\prime}$ and $X^{\prime} \approx_{S} Y^{\prime}$.

Proof. Let $X_{1}, \ldots, X_{n}$ be the formal variables of $S$,

$S=\left\{X_{1}=F_{X_{1}}, \ldots, X_{n}=F_{X_{n}}\right\}, D_{X_{1}}, \ldots, D_{X_{n}}$ be a set of provable solutions of $S$ for $X_{1}, \ldots, X_{n}$ respectively, i.e. $\vdash D_{X_{i}}=F_{X_{i}}\left\{D_{X_{i}} / X_{i} \mid i=1, \ldots, n\right\}$ holds for $i=1, \ldots, n$ (according to Theorem 5.2 there exist such solutions). Without loss of generality let $X, Y$ be $X_{1}$ and $X_{2}$ respectively, thus $X_{1}$ is a bottom variable. In particular, $D_{X_{1}}={ }_{b}^{\Delta} F_{X_{1}}\left\{D_{X_{i}} / X_{i} \mid i=1, \ldots, n\right\}$ and

$D_{X_{2}}={ }_{b}^{\Delta} F_{X_{2}}\left\{D_{X_{i}} / X_{i} \mid i=1, \ldots, n\right\}$ follow from the soundness of the inference system, and the condition $X_{1} \approx_{S} X_{2}$ forces $D_{X_{1}} \approx_{b}^{\Delta} D_{X_{2}}$ to hold, thus

$F_{X_{1}}\left\{D_{X_{i}} / X_{i} \mid i=1, \ldots, n\right\} \approx_{b}^{\Delta} F_{X_{2}}\left\{D_{X_{i}} / X_{i} \mid i=1, \ldots, n\right\}$.

First we prove the following basic fact: whenever $F_{X_{1}}\left\{D_{X_{i}} / X_{i} \mid i=1, \ldots, n\right\} \Longrightarrow G$ with $G \approx_{b}^{\Delta} D_{X_{1}}$, then $G \equiv F_{X_{1}}\left\{D_{X_{i}} / X_{i} \mid i=1, \ldots, n\right\}$. We can prove this by induction on the length of the $\stackrel{\tau}{\longrightarrow}$ transition sequence from $F_{X_{1}}\left\{D_{X_{i}} / X_{i} \mid i=1, \ldots, n\right\}$ to $G$. If the length is zero, then $G$ does not make a move from $F_{X_{1}}\left\{D_{X_{i}} / X_{i} \mid i=1, \ldots, n\right\}$, clearly in this case $G \equiv F_{X_{1}}\left\{D_{X_{i}} / X_{i} \mid i=1, \ldots, n\right\}$ holds. If the length is not zero, then there is $G^{\prime}$ such that $F_{X_{1}}\left\{D_{X_{i}} / X_{i} \mid i=1, \ldots, n\right\} \stackrel{\tau}{\longrightarrow} G^{\prime}, G^{\prime} \Longrightarrow G$ and the number of transitions from $G^{\prime}$ to $G$ is one less than that from $F_{X_{1}}\left\{D_{X_{i}} / X_{i} \mid i=1, \ldots, n\right\}$, and since $D_{X_{1}}$ provably solves $S, D_{X_{1}}={ }_{b}^{\Delta} F_{X_{1}}\left\{D_{X_{i}} / X_{i} \mid i=1, \ldots, n\right\}$ follows from the soundness of the inference system, thus

$F_{X_{1}}\left\{D_{X_{i}} / X_{i} \mid i=1, \ldots, n\right\} \approx_{b}^{\Delta} D_{X_{1}} \approx_{b}^{\Delta} G$, we know from stutter lemma that $G^{\prime} \approx_{b}^{\Delta} G \approx_{b}^{\Delta} D_{1}$. On the other hand, the form of the expression $F_{X_{1}}$ determines that there are two possible ways for $F_{X_{1}}\left\{D_{1} / X_{1}, \ldots, D_{n} / X_{n}\right\}$ to make a $\stackrel{\tau}{\longrightarrow}$ transition to $G^{\prime}$ : either for some $k$ such that $F_{X_{1}} \stackrel{\tau}{\longrightarrow} X_{k}$ and $G^{\prime}$ is $D_{k}$, or $F_{X_{1}}$ is a loop expression and $G^{\prime} \equiv F_{X_{1}}\left\{D_{X_{i}} / X_{i} \mid i=1, \ldots, n\right\}$. Since $X_{1}$ is a bottom variable, $X_{1} \tilde{\nabla} X_{k}$ would force $X_{k} \not_{S} X_{1}$, and then $G^{\prime} \equiv D_{k} \not \varpi_{b}^{\Delta} D_{1}$, so the first choice is not possible. Then it must be the second choice, and in this case $G^{\prime}$ is $F_{X_{1}}\left\{D_{X_{i}} / X_{i} \mid i=1, \ldots, n\right\}$, and there is a shorter sequence of $\stackrel{\tau}{\longrightarrow}$ transitions to $G$, by the induction hypothesis in this case $G \equiv F_{X_{1}}\left\{D_{X_{i}} / X_{i} \mid i=1, \ldots, n\right\}$.

With this basic fact, we proceed to prove 2) as follows. 1) can be proved in the same way. Suppose $F_{X_{2}} \stackrel{a}{\longrightarrow} X_{k}$ where $a \neq \tau$ or $X_{k} \not_{S} X_{2}$. Thus $a=\tau$ or $D_{X_{k}} \not_{b}^{\Delta} D_{X_{2}}$, and
$F_{X_{2}}\left\{D_{X_{i}} / X_{i} \mid i=1 \ldots, n\right\} \stackrel{a}{\longrightarrow} D_{X_{k}}$. Then there must exist $G, G^{\prime}$ such that $F_{X_{1}}\left\{D_{X_{i}} / X_{i} \mid i=1 \ldots, n\right\} \Longrightarrow G, G \stackrel{a}{\longrightarrow} G^{\prime}$ such that $G \approx_{b}^{\Delta} D_{X_{1}}$ and $G^{\prime} \approx_{b}^{\Delta} D_{X_{i}}$. By the property proved above, it must be that $G \equiv F_{X_{1}}\left\{D_{X_{i}} / X_{i} \mid i=1, \ldots, n\right\}$, thus $F_{X_{1}}\left\{D_{X_{i}} / X_{i} \mid i=1, \ldots, n\right\} \stackrel{a}{\longrightarrow} G^{\prime}$, and the form of the expression $F_{X_{1}}$ determines that there are two possible ways for $F_{X_{1}}\left\{D_{1} / X_{1}, \ldots, D_{n} / X_{n}\right\}$ to make a $\stackrel{a}{\longrightarrow}$ transition to $G^{\prime}$ : either for some $l$ such that $F_{X_{1}} \stackrel{a}{\longrightarrow} X_{l}$ and $G^{\prime}$ is $D_{X_{l}}$, or $a=\tau$ and $F_{X_{1}}$ is a loop expression and $G^{\prime}$ is

$F_{X_{1}}\left\{D_{X_{i}} / X_{i} \mid i=1, \ldots, n\right\}$. The second alternative is not possible, since that would imply $D_{X_{k}} \approx_{b}^{\Delta} G^{\prime} \approx_{b}^{\Delta} D_{X_{2}}$ and $a=\tau$, contradicts the condition that $a \neq \tau$ or $X_{k} \not \varpi_{S} X_{2}$. Thus we found $X_{l}$ such that $F_{X_{1}} \stackrel{a}{\longrightarrow} X_{l}$, and since $D_{X_{l}}$ provably solves $X_{l}$ and $D_{X_{l}} \approx_{b}^{\Delta} D_{X_{k}}$, hence $X_{l} \approx_{S} X_{k}$.

Lemma 5.8: Let $S$ be an SES, $X$ be a bottom variable with $X=F_{X} \in S$. If $F_{X}$ is not a loop expression, then whenever $Y=F_{Y} \in S$ with $X \approx_{S} Y, F_{Y}$ is not a loop expression.

Proof. Any solution to a bottom variable $X$ with $X=F_{X} \in S$ where $F_{X}$ is not a loop expression must not be divergent, while any solution to a variable $Y$ (bottom or not) with $Y=F_{Y} \in S$ where $F_{Y}$ is a loop expression must be divergent, thus in this case $X \not \not_{S} Y$.

Definition 5.9: Let $S$ be an SES with the set of formal variables $V, X=F_{X}$ be an equation in $S$. Define $F_{X}^{0}$ and $F_{X}^{1}$, called the stuttering derivative and non stuttering derivative of $X$ in $S$ respectively, as the follows

$F_{X}^{0}=\Sigma\left\{\tau . Y \mid F_{X} \stackrel{\tau}{\longrightarrow} Y, X \approx_{S} Y\right\}$, $F_{X}^{1}=\Sigma\left\{a . Y \mid F_{X} \stackrel{a}{\longrightarrow} Y, a \neq \tau\right.$ or $\left.X \nsim_{S} Y\right\}+\Sigma\left\{W \mid F_{X} \triangleright W\right\}$.

Intuitively, $F_{X}^{0}$ is a sum which consists of the $\tau$-derivatives of $F_{X}$ which do not change equivalence class, while $F_{X}^{1}$ is a sum which consists of the rest of the derivatives plus the free variables. Here are some simple properties of derivatives.

Proposition 5.10: Let $S$ be an SES with formal variables in $V, X=F_{X}, X^{\prime}=F_{X^{\prime}}$ be equations in $S,\left\{E_{Y} / Y \mid Y \in V\right\}$ be a $\approx_{S}$-respecting substitution on $V$ s.t. whenever $Y \approx_{S} Y^{\prime}$ then $E_{Y} \equiv E_{Y^{\prime}}$.

1) if $F_{X}$ is not a loop expression, then

$\vdash F_{X}\left\{E_{Y} / Y \mid Y \in V\right\}$

$=F_{X}^{0}\left\{E_{Y} / Y \mid Y \in V\right\}+F_{X}^{1}\left\{E_{Y} / Y \mid Y \in V\right\} ;$

2) if $F_{X}$ is a loop expression, then

$\vdash F_{X}\left\{E_{Y} / Y \mid Y \in V\right\}$

$=\tau^{*}\left(F_{X}^{0}\left\{E_{Y} / Y \mid Y \in V\right\}+F_{X}^{1}\left\{E_{Y} / Y \mid Y \in V\right\}\right)$;

3) $\vdash F_{X}\left\{E_{Y} / Y \mid Y \in V\right\}$

$=F_{X}\left\{E_{Y} / Y \mid Y \in V\right\}+F_{X}^{1}\left\{E_{Y} / Y \mid Y \in V\right\}$;

4) if $X$ is a bottom variable then

$\vdash F_{X}^{0}\left\{E_{Y} / Y \mid Y \in V\right\}=\mathbf{0}$, otherwise

$\vdash F_{X}^{0}\left\{E_{Y} / Y \mid Y \in V\right\}=\tau . E_{X}$;

5) if $X \approx_{S} X^{\prime}$, and $X$ is a bottom variable then

$\vdash F_{X}^{1}\left\{E_{Y} / Y \mid Y \in V\right\}$

$=F_{X}^{1}\left\{E_{Y} / Y \mid Y \in V\right\}+F_{X^{\prime}}^{1}\left\{E_{Y} / Y \mid Y \in V\right\}$.

Proof. To prove 1), note that when $F_{X}$ is not a loop expression then $F_{X}^{0}+F_{X}^{1}$ and $F_{X}$ are two sums with the same set summands. Thus $F_{X}^{0}\left\{E_{Y} / Y \mid Y \in V\right\}+F_{X}^{1}\left\{E_{Y} / Y \mid Y \in V\right\}$ and $F_{X}\left\{E_{Y} / Y \mid Y \in V\right\}$ are also two sums with the same set of summands, so by S1-S4 
$\vdash F_{X}\left\{E_{Y} / Y \mid Y \in V\right\}$

$=F_{X}^{0}\left\{E_{Y} / Y \mid Y \in V\right\}+F_{X}^{1}\left\{E_{Y} / Y \mid Y \in V\right\}$.

2) can be proved in the same way.

3) easily follows from 1) and 2) by using S3 and D2.

To see 4), first note that by Lemma 5.6 $F_{X}^{0}$ is an empty sum when $X$ is a bottom variable. Thus $F_{X}^{0}\left\{E_{Y} / Y \mid Y \in V\right\}$ is also an empty sum, and $\vdash F_{X}^{0}\left\{E_{Y} / Y \mid Y \in V\right\}=\mathbf{0}$. When $X$ is not a bottom element, then there exists $X^{\prime}$ such that $X \approx_{S} X^{\prime}$ and $F_{X} \stackrel{\tau}{\longrightarrow} X^{\prime}$. Then by the $\approx_{S}$-respecting property of the substitution, for all such $X^{\prime}$ it holds that $E_{X^{\prime}} \equiv E_{X}$, hence $\vdash F_{X}^{0}\left\{E_{Y} / Y \mid Y \in V\right\}=\tau$. $E_{X}$.

To prove 5), note that, according to Lemma 5.7 when $X \approx_{S} X^{\prime}$ and $X$ is a bottom variable, with the $\approx_{S^{-}}$ respecting condition of the substitution, any summand of $F_{X^{\prime}}^{1}\left\{E_{Y} / Y \mid Y \in V\right\}$ is a summand of $F_{X}^{1}\left\{E_{Y} / Y \mid Y \in V\right\}$. Thus by $\mathrm{S} 3$

$\vdash F_{X}^{1}\left\{E_{Y} / Y \mid Y \in V\right\}$

$=F_{X}^{1}\left\{E_{Y} / Y \mid Y \in V\right\}+F_{X^{\prime}}^{1}\left\{E_{Y} / Y \mid Y \in V\right\}$.

Definition 5.11: For a recursive equation set $S$, define a new recursive equation set $\tau(S)=\left\{X=\tau \cdot F_{X} \mid X=F_{X} \in S\right\}$.

Then $\tau(S)$ has the same set of formal variables as $S$.

Lemma 5.12: Let $S$ be a recursive equation set, $X, Y$ be formal variables of $S$. Then $X \tilde{\triangleright}_{S} Y$ if and only if $X \tilde{\triangleright}_{\tau(S)} Y$.

Proof. Obvious.

Lemma 5.13: Let $S$ be an SES. Then $\tau(S)$ is guarded.

Proof. Note that an SES is a guarded recursive equation set, then the claim immediately follows from Lemma 5.12

Proposition 5.14: Let $S$ be an SES, $X$ be one of the formal variables. If $E \in \mathcal{E}$ is a provable solution of $S$ for $X$ then $\tau$.E is a provable solution of $\tau(S)$ for $X$.

Proof. It easily follows from T1 (Theorem 3.6).

We need the following lemma about successive substitution applied on a simple sum $F$ (Definition 4.6). The lemma also holds for general expression $F$, which is the well-known substitution lemma (for example Lemma 2.1 in [9]). The simplified version is sufficient for our purpose, and is easy to establish.

Lemma 5.15: Let $F$ be a simple sum, $X_{1}, \ldots, X_{n}$ be $n$ variables, $E_{1}, \ldots, E_{n}$ be $n$ expressions, $Z_{1}, \ldots, Z_{m}$ be $m$ variables which do not occur in $F, N_{1}, \ldots, N_{m}$ be $m$ expressions. Then

$F\left\{E_{1} / X_{1}, \ldots, E_{n} / X_{n}\right\}\left\{N_{1} / Z_{1}, \ldots, N_{m} / Z_{m}\right\} \equiv$

$F\left\{E_{1}\left\{N_{1} / Z_{1}, \ldots, N_{m} / Z_{m}\right\} / X_{1}\right.$,

$\left.\ldots, E_{n}\left\{N_{1} / Z_{1}, \ldots, N_{m} / Z_{m}\right\} / X_{n}\right\}$

Proof. Can be proved by an easy induction on the number of summands in $F$.

We now arrive at the main result of this paper, we name it quotioning theorem because the proof uses the quotient construction of an SES.

Theorem 5.16: (Quotienting) Let $S$ be an SES, $X, X^{\prime}$ be two formal variables of $S$. If $X \approx_{S} X^{\prime}$, then $\tau(S)$ has common provable solution for $X$ and $X^{\prime}$.

Proof. Let $V$ be the set of formal variables of $S$. Since $S$ is an SES which is a guarded recursive equation system, $\approx_{S}$ is an equivalence relation on $V$, we can assume that $\approx_{S}$ partitions $V$ into $n$ equivalence classes $C_{1}, \ldots, C_{n}$. According to Lemma
5.7 there exists a bottom variable in each equivalence class, thus we can assume that $X_{1}, \ldots, X_{n}$ are $n$ designated bottom variables such that $X_{i} \in C_{i}$ with $X_{i}=F_{X_{i}} \in S$ for $i=1, \ldots, n$. For $X \in V$, we define the index of $X$, written $\iota(X)$, such that $\iota(X)=i$ if $X \in C_{i}$. Let $Z_{1}, \ldots, Z_{n}$ be $n$ variables which are not variables occurring in any equation in $S$. We construct the following $\approx_{S}$-quotient equation system of $S$ :

$$
S / \approx_{S}=\left\{Z_{i}=G_{i} \mid i=1, \ldots, n\right\}
$$

where $G_{i} \equiv F_{X_{i}}\left\{Z_{\iota(Y)} / Y \mid Y \in V\right\}$. Then $S / \approx_{S}$ is a recursive equation system, and according to Theorem 5.2 , there exist $n$ expressions $B_{1}, \ldots, B_{n}$ such that for $i=1, \ldots, n$ we have the following equality:

$$
\vdash B_{i}=G_{i}\left\{B_{1} / Z_{1}, \ldots, B_{n} / Z_{n}\right\} .
$$

With this equality, we have that for each $i \in\{1, \ldots, n\}$ :

$\vdash B_{i}=G_{i}\left\{B_{1} / Z_{1}, \ldots, B_{n} / Z_{n}\right\}$

$=F_{X_{i}}\left\{Z_{\iota(Y)} / Y \mid Y \in V\right\}\left\{B_{1} / Z_{1}, \ldots, B_{n} / Z_{n}\right\}$ def.of $G_{i}$

$=F_{X_{i}}\left\{Z_{\iota(Y)}\left\{B_{1} / Z_{1}, \ldots, B_{n} / Z_{n}\right\} / Y \mid Y \in V\right\}$ Lemma5.15

$=F_{X_{i}}\left\{B_{\iota(Y)} / Y \mid Y \in V\right\}$

Thus for $i=1, \ldots, n$ we proved the following equality which will be used later

$$
\vdash B_{i}=F_{X_{i}}\left\{B_{\iota(Y)} / Y \mid Y \in V\right\} .
$$

Our next step is to prove that for each $X \in V$ with $X=F_{X} \in S$ it holds that

$\vdash \tau . F_{X}\left\{B_{\iota(Y)} / Y \mid Y \in V\right\}=\tau . F_{X_{i}}\left\{B_{\iota(Y)} / Y \mid Y \in V\right\}$,

where $i=\iota(X)$, i.e. $X_{i}$ is the designated bottom variable in $[X]$ and $X_{i}=F_{X_{i}} \in S$. To prove this we discuss four cases according to whether $X$ is a bottom variable and whether $F_{X}$ is a loop expression by using Proposition 5.10 (note that $\left\{B_{\iota(Y)} / Y \mid Y \in V\right\}$ is clearly a $\approx_{S}$-respecting substitution).

If $X$ is a bottom variable and $F_{X}$ is not a loop expression, then by Lemma 5.8 $F_{X_{i}}$ is not a loop expression. So $\vdash \tau . F_{X}\left\{B_{\iota(Y)} / Y \mid Y \in V\right\}$

$=\tau .\left(F_{X}^{0}\left\{B_{\iota(Y)} / Y \mid Y \in V\right\}+F_{X}^{1}\left\{B_{\iota(Y)} / Y \mid Y \in V\right\}\right)$

1) of proposition 5.10

$=\tau . F_{X}^{1}\left\{B_{\iota(Y)} / Y \mid Y \in V\right\} \quad$ 4) of prop. 5.10 and S4

$=\tau \cdot\left(F_{X}^{1}\left\{B_{\iota(Y)} / Y \mid Y \in V\right\}+F_{X_{i}}^{1}\left\{B_{\iota(Y)} / Y \mid Y \in V\right\}\right)$

5) of proposition 5.10, $X$ is a bottom variable

$=\tau . F_{X_{i}}^{1}\left\{B_{\iota(Y)} / Y \mid Y \in V\right\} \quad$ 5) of prop. 5.10, $X_{i}$ a bot. var.

$=\tau \cdot\left(F_{X_{i}}^{0}\left\{B_{\iota(Y)} / Y \mid Y \in V\right\}+F_{X_{i}}^{1}\left\{B_{\iota(Y)} / Y \mid Y \in V\right\}\right)$

4) of prop. 5.10 and $S 4$

$=\tau . F_{X_{i}}\left\{B_{\iota(Y)} / Y \mid Y \in V\right\}$

1) of proposition 5.10

If $X$ is a bottom variable and $F_{X}$ is a loop expression, then $F_{X_{i}}$ is also a loop expression (otherwise, since $X_{i}$ is a bottom variable, by Lemma $5.8 F_{X}$ is not a loop expression). Then $\vdash \tau . F_{X}\left\{B_{\iota(Y)} / Y \mid Y \in V\right\}$

$=\tau \cdot \tau^{*}\left(F_{X}^{0}\left\{B_{\iota(Y)} / Y \mid Y \in V\right\}+F_{X}^{1}\left\{B_{\iota(Y)} / Y \mid Y \in V\right\}\right)$

2) of proposition 5.10

$=\tau \cdot \tau^{*} F_{X}^{1}\left\{B_{\iota(Y)} / Y \mid Y \in V\right\} \quad$ 4) of prop. 5.10 and $\mathrm{S} 4$

$=\tau \cdot \tau^{*}\left(F_{X}^{1}\left\{B_{\iota(Y)} / Y \mid Y \in V\right\}+F_{X_{i}}^{1}\left\{B_{\iota(Y)} / Y \mid Y \in V\right\}\right)$

5) of proposition 5.10, $X$ is a bottom variable $=\tau \cdot \tau^{*} F_{X_{i}}^{1}\left\{B_{\iota(Y)} / Y \mid Y \in V\right\}$ 5) of prop. 5.10, $X_{i}$ bot. var. 
$=\tau \cdot \tau^{*}\left(F_{X_{i}}^{0}\left\{B_{\iota(Y)} / Y \mid Y \in V\right\}+F_{X_{i}}^{1}\left\{B_{\iota(Y)} / Y \mid Y \in V\right\}\right)$

4) of prop. 5.10 and $\mathrm{S} 4$

$=\tau \cdot F_{X_{i}}\left\{B_{\iota(Y)} / Y \mid Y \in V\right\}$

2) of proposition 5.10

If $X$ is not a bottom variable and $F_{X}$ is a loop expression, then $F_{X_{i}}$ is also a loop expression (otherwise, since $X_{i}$ is a bottom variable, $F_{X}$ cannot be a loop expression). Now

$\vdash \tau . F_{X}\left\{B_{\iota(Y)} / Y \mid Y \in V\right\}$

$=\tau \cdot \tau^{*}\left(F_{X}^{0}\left\{B_{\iota(Y)} / Y \mid Y \in V\right\}+F_{X}^{1}\left\{B_{\iota(Y)} / Y \mid Y \in V\right\}\right)$

2) of proposition 5.10

$=\tau \cdot \tau^{*}\left(\tau \cdot B_{\iota(X)}+F_{X}^{1}\left\{B_{\iota(Y)} / Y \mid Y \in V\right\}\right)$ 4) of prop. 5.10

$=\tau \cdot \tau^{*}\left(\tau \cdot B_{i}+F_{X}^{1}\left\{B_{\iota(Y)} / Y \mid Y \in V\right\}\right)$

identity

$=\tau \cdot \tau^{*}\left(\tau . F_{X_{i}}\left\{B_{\iota(Y)} / Y \mid Y \in V\right\}+F_{X}^{1}\left\{B_{\iota(Y)} / Y \mid Y \in V\right\}\right)$ (2)

$=\tau \cdot \tau^{*}\left(\tau \cdot \tau^{*}\left(F_{X_{i}}^{0}\left\{B_{\iota(Y)} / Y \mid Y \in V\right\}+F_{X_{i}}^{1}\left\{B_{\iota(Y)} / Y \mid Y \in V\right\}\right)\right.$

$\left.+F_{X}^{1}\left\{B_{\iota(Y)} / Y \mid Y \in V\right\}\right) \quad 2$ ) of proposition 5.10

$=\tau \cdot \tau^{*}\left(\tau \cdot \tau^{*} F_{X_{i}}^{1}\left\{B_{\iota(Y)} / Y \mid Y \in V\right\}\right.$

$\left.+F_{X}^{1}\left\{B_{\iota(Y)} / Y \mid Y \in V\right\}\right)$

4) of proposition 5.10

$=\tau \cdot \tau^{*}\left(\tau \cdot \tau^{*}\left(F_{X_{i}}^{1}\left\{B_{\iota(Y)} / Y \mid Y \in V\right\}+F_{X}^{1}\left\{B_{\iota(Y)} / Y \mid Y \in V\right\}\right)\right.$

$\left.+F_{X}^{1}\left\{B_{\iota(Y)} / Y \mid Y \in V\right\}\right) \quad$ 5) of proposition 5.10

$=\tau \cdot\left(\tau \cdot \tau^{*}\left(F_{X_{i}}^{1}\left\{B_{\iota(Y)} / Y \mid Y \in V\right\}+F_{X}^{1}\left\{B_{\iota(Y)} / Y \mid Y \in V\right\}\right)\right.$

$\left.+F_{X}^{1}\left\{B_{\iota(Y)} / Y \mid Y \in V\right\}\right)$

D5

$=\tau \cdot\left(\tau \cdot\left(\tau^{*}\left(F_{X_{i}}^{1}\left\{B_{\iota(Y)} / Y \mid Y \in V\right\}+F_{X}^{1}\left\{B_{\iota(Y)} / Y \mid Y \in V\right\}\right)\right.\right.$

$\left.\left.+F_{X}^{1}\left\{B_{\iota(Y)} / Y \mid Y \in V\right\}\right)+F_{X}^{1}\left\{B_{\iota(Y)} / Y \mid Y \in V\right\}\right) \quad$ D2

$=\tau \cdot\left(\tau^{*}\left(F_{X_{i}}^{1}\left\{B_{\iota(Y)} / Y \mid Y \in V\right\}+F_{X}^{1}\left\{B_{\iota(Y)} / Y \mid Y \in V\right\}\right)\right.$

$\left.+F_{X}^{1}\left\{B_{\iota(Y)} / Y \mid Y \in V\right\}\right)$

$=\tau \cdot \tau^{*}\left(F_{X_{i}}^{1}\left\{B_{\iota(Y)} / Y \mid Y \in V\right\}+F_{X}^{1}\left\{B_{\iota(Y)} / Y \mid Y \in V\right\}\right) \mathrm{D} 2$

$=\tau \cdot \tau^{*} F_{X_{i}}^{1}\left\{B_{\iota(Y)} / Y \mid Y \in V\right\} \quad$ 5) of proposition 5.10

$=\tau \cdot \tau^{*}\left(F_{X_{i}}^{0}\left\{B_{\iota(Y)} / Y \mid Y \in V\right\}+F_{X_{i}}^{1}\left\{B_{\iota(Y)} / Y \mid Y \in V\right\}\right)$

$=\tau \cdot F_{X_{i}}\left\{B_{\iota(Y)} / Y \mid Y \in V\right\} \quad$ 2) of proposition 5.10

If $X$ is not a bottom variable and $F_{X}$ is not a loop expression, then

$\vdash \tau . F_{X}\left\{B_{\iota(Y)} / Y \mid Y \in V\right\}$

$=\tau \cdot\left(F_{X}^{0}\left\{B_{\iota(Y)} / Y \mid Y \in V\right\}+F_{X}^{1}\left\{B_{\iota(Y)} / Y \mid Y \in V\right\}\right)$

1) of proposition 5.10

$=\tau \cdot\left(\tau \cdot B_{\iota(X)}+F_{X}^{1}\left\{B_{\iota(Y)} / Y \mid Y \in V\right\}\right) \quad$ 4) of prop. 5.10

$=\tau \cdot\left(\tau . B_{i}+F_{X}^{1}\left\{B_{\iota(Y)} / Y \mid Y \in V\right\}\right)$ identity

$=\tau \cdot\left(\tau \cdot F_{X_{i}}\left\{B_{\iota(Y)} / Y \mid Y \in V\right\}+F_{X}^{1}\left\{B_{\iota(Y)} / Y \mid Y \in V\right\}\right)$

$=\tau \cdot\left(\tau \cdot\left(F_{X_{i}}\left\{B_{\iota(Y)} / Y \mid Y \in V\right\}+F_{X_{i}}^{1}\left\{B_{\iota(Y)} / Y \mid Y \in V\right\}\right)\right.$

$\left.\left.+F_{X}^{1}\left\{B_{\iota(Y)} / Y \mid Y \in V\right\}\right) \quad 3\right)$ of proposition 5.10

$=\tau .\left(\tau .\left(F_{X_{i}}\left\{B_{\iota(Y)} / Y \mid Y \in V\right\}+F_{X_{i}}^{1}\left\{B_{\iota(Y)} / Y \mid Y \in V\right\}\right.\right.$

$\left.\left.+F_{X}^{1}\left\{B_{\iota(Y)} / Y \mid Y \in V\right\}\right)+F_{X}^{1}\left\{B_{\iota(Y)} / Y \mid Y \in V\right\}\right)$

5) of proposition. 5.10

$=\tau \cdot\left(F_{X_{i}}\left\{B_{\iota(Y)} / Y \mid Y \in V\right\}+F_{X_{i}}^{1}\left\{B_{\iota(Y)} / Y \mid Y \in V\right\}\right)$

$\left.+F_{X}^{1}\left\{B_{\iota(Y)} / Y \mid Y \in V\right\}\right)$

$\left.=\tau \cdot F_{X_{i}}\left\{B_{\iota(Y)} / Y \mid Y \in V\right\} \quad 3\right)$ and 5) of proposition 5.10

With this, we finished the discussion of all four cases.

To conclude the proof, for each $X=F_{X} \in S$ with $\iota(X)=i$ we have

$\vdash \tau . B_{\iota(X)}=\tau . B_{i}$

$=\tau \cdot F_{X_{i}}\left\{B_{\iota(Y)} / Y \mid Y \in V\right\}$

identity

$=\tau . F_{X}\left\{B_{\iota(Y)} / Y \mid Y \in V\right\}$

$=\tau . F_{X}\left\{\tau \cdot B_{\iota(Y)} / Y \mid Y \in V\right\}$.

So $\tau B_{u(X)}$ is a provable solution to $\tau(S)$ for $X$. If $X \approx_{S} \frac{3.6}{X^{\prime}}$ then $\tau . B_{\iota\left(X^{\prime}\right)}$ is a provable solution to $\tau(S)$ for $X^{\prime}$, but in this case $\iota(X)=\iota\left(X^{\prime}\right)$, hence $\tau \cdot B_{\iota(X)} \equiv \tau \cdot B_{\iota\left(X^{\prime}\right)}$, so $\tau(S)$ has common provable solution for $X$ and $X^{\prime}$.

\section{The Completeness Proof}

Now we are prepared to prove the completeness of the axiomatisation.

Theorem 6.1: Let $E \in \mathcal{E}$. If $E$ is guarded, then there is a standard equation system $S$ with a formal variable $X$, such that $E$ is a provable solution of $S$ for $X$.

Proof. It is proved by induction on the structure of $E$.

(i) $E \equiv \mathbf{0}$. Take $S$ to be the single equation $X=\mathbf{0}$.

(ii) $E \equiv W$. Take $S$ to be the single equation $X=W$.

(iii) $E \equiv a . E^{\prime}$. By the induction hypothesis $E^{\prime}$ provably solves a standard equation set $S^{\prime}$ for variable $X^{\prime}$. Then add the equation $X=a . X^{\prime}$ to $S^{\prime}$ to form $S, E$ provably solves $S$ for $X$, and $S$ is a standard equation system.

(iv) $E \equiv E^{\prime}+E^{\prime \prime}$. By the induction hypothesis $E^{\prime}$ provably solves a standard equation set $S^{\prime}$ for $X^{\prime}$ with $X^{\prime}=F^{\prime} \in S^{\prime}$, and $E^{\prime \prime}$ provably solves a standard equation set $S^{\prime \prime}$ for $X^{\prime \prime}$ with $X^{\prime \prime}=F^{\prime \prime} \in S^{\prime \prime}$ (assume that the formal variables of $S^{\prime}$ are distinct from those of $S^{\prime \prime}$ ). There are four cases to discuss according to the forms of $F^{\prime}$ and $F^{\prime \prime}$. If both $F^{\prime}$ and $F^{\prime \prime}$ are standard sums then take $S^{\prime} \cup S^{\prime \prime}$ and add $X=F^{\prime}+F^{\prime \prime}$ to form $S$ (with $X$ distinct from the formal and free variables of $S^{\prime}$ and $\left.S^{\prime \prime}\right)$. If $F^{\prime}$ is a standard sum while $F^{\prime \prime} \equiv \tau^{*} G^{\prime \prime}$ for some standard sum $G^{\prime \prime}$, then take $S^{\prime} \cup S^{\prime \prime}$ and add the equation $X=F^{\prime}+\tau \cdot X^{\prime \prime}+G^{\prime \prime}$ to form $S$ (with $X$ distinct from the formal and free variables of $S^{\prime}$ and $S^{\prime \prime}$ ). Likewise for the case where $F^{\prime \prime}$ is a standard sum while $F^{\prime}$ is in loop form. If $F^{\prime} \equiv \tau^{*} G^{\prime}$ and $F^{\prime \prime} \equiv \tau^{*} G^{\prime \prime}$ for standard sums $G^{\prime}$ and $G^{\prime \prime}$, then take $S^{\prime} \cup S^{\prime \prime}$ and add $X=\tau \cdot X^{\prime}+G^{\prime}+\tau \cdot X^{\prime \prime}+G^{\prime \prime}$ to form $S$ (with $X$ distinct from the formal and free variables of $S^{\prime}$ and $\left.S^{\prime \prime}\right)$. With D1 it is easy to see that in all the cases $S$ is a standard equation set and that $E$ provably solves $S$ for $X$.

(v) $E \equiv \mu W^{\prime} . E^{\prime}$, with $W^{\prime}$ guarded in $E^{\prime}$. By the induction hypothesis $E^{\prime}$ provably solves a standard equation set $S^{\prime}$ for variable $X^{\prime}$ with $X^{\prime}=H \in S^{\prime}$. We discuss two cases according to whether $H$ is a standard sum or not. If $H$ is a standard sum, take $\left\{Y=G\left\{H / W^{\prime}\right\} \mid Y=G \in S^{\prime}\right\}$ to form $S$, and if $H \equiv \tau^{*} H^{\prime}$ where $H^{\prime}$ is a standard sum, take $\left\{Y=G\left\{\tau \cdot X^{\prime}+H^{\prime} / W^{\prime}\right\} \mid Y=G \in S^{\prime}\right\}$ to form $S$. Since $W^{\prime}$ is not a formal variable of $S^{\prime}$, in both cases $S$ is a standard equation set. Also $E$ provably solves $S$ for $X$ (in the second case D1 is used to show this).

(vi) $E \equiv \tau^{*} E^{\prime}$. By the induction hypothesis $E^{\prime}$ provably solves a standard equation set $S^{\prime}$ for $X^{\prime}$ with $X^{\prime}=F^{\prime} \in S^{\prime}$. If $F^{\prime}$ is a standard sum, then add the equation $X=\tau^{*} F^{\prime}$ to $S^{\prime}$ to form $S, E$ provably solves $S$ for $X$, and $S$ is a standard equation system. If $F^{\prime} \equiv \tau^{*} F^{\prime \prime}$ where $F^{\prime \prime}$ is a standard sum, then add the equation $X=F^{\prime}$ to form $S$. According to D6, $E$ provably solves $S$, and $S$ is a standard equation set.

Lemma 6.2: (Promotion) Let $E, F \in \mathcal{E}$ be guarded expressions. If $E \approx_{b}^{\Delta} F$, then $\vdash \tau$. $E=\tau$.F.

Proof. According to Theorem 6.1, there exist standard equation systems $S_{1}$ with a formal variable $X$ and $S_{2}$ with a formal variable $Y$ (assume they have disjoint sets of formal variables) such that $E$ and $F$ provably solve $S_{1}$ for variable $X$ and $S_{2}$ for variable $Y$ respectively. Then it is clear that $S_{1} \cup S_{2}$ is an SES 
with formal variables $X$ and $Y$, and that $E$ and $F$ provably solve $S_{1} \cup S_{2}$ for $X$ and $Y$ respectively. Then according to Proposition 5.14 $\tau$.E and $\tau . F$ provably solve $\tau\left(S_{1} \cup S_{2}\right)$ for $X$ and $Y$ respectively. If $E \approx_{b}^{\Delta} F$, then $X \approx_{S_{1} \cup S_{2}} Y$, and according to Theorem 5.16 $X$ and $Y$ have common solution in $\tau\left(S_{1} \cup S_{2}\right)$, i.e. there is an expression $B$ which provably solves $\tau\left(S_{1} \cup S_{2}\right)$ for $X$ as well as for $Y$. Since $S_{1} \cup S_{2}$ is an SES which is guarded, by Lemma5.13 $\tau\left(S_{1} \cup S_{2}\right)$ is also guarded, according to Theorem 5.2 it has unique solution. Now both $\tau$. $E$ and $B$ provably solve $\tau\left(S_{1} \cup S_{2}\right)$ for $X$ so $\vdash \tau$. $E=B$, and both $\tau . F$ and $B$ provably solve $\tau\left(S_{1} \cup S_{2}\right)$ for $Y$ so $\vdash \tau . F=B$, hence $\vdash \tau$. $E=\tau$.F.

Theorem 6.3: Let $E, F \in \mathcal{E}$. If $E={ }_{b}^{\Delta} F$ then $\vdash E=F$.

Proof. First we show that in this case $\vdash E+F=F$. By Theorem 4.7 there exist guarded expressions $E_{1}, \ldots, E_{n}$, and variables $W_{1}, \ldots, W_{m}$, such that

$$
\vdash E=\sum_{i=1}^{n} a_{i} . E_{i}+\sum_{j=1}^{m} W_{j} .
$$

Thus in order to prove $\vdash E+F=F$ we only need to show that $\vdash \sum_{i=1}^{n} a_{i} \cdot E_{i}+\sum_{j=1}^{m} W_{j}+F=F$, and we will do this by induction on $m+n$. If $m+n=0$, by S4 obviously this holds. If $m>0$, then $\sum_{i=1}^{n} a_{i} . E_{i}+\sum_{j=1}^{m} W_{j} \triangleright W_{m}$. Since $\sum_{i=1}^{n} a_{i} \cdot E_{i}+\sum_{j=1}^{m} W_{j}={ }_{b}^{\Delta} E={ }_{b}^{\Delta} F$ by $(A)$ and the soundness of the proof system and $E={ }_{b}^{\Delta} F$, it follows that $F \triangleright W_{m}$. Thus $\vdash F=F+W_{m}$ follows from Lemma 3.7. Now

$\vdash \sum_{i=1}^{n} a_{i} \cdot E_{i}+\sum_{j=1}^{m} W_{j}+F$

$=\sum_{i=1}^{n} a_{i} \cdot E_{i}+\sum_{j=1}^{m-1} W_{j}+F+W_{m}$

$=\sum_{i=1}^{n} a_{i} \cdot E_{i}+\sum_{j=1}^{m-1} W_{j}+F$

$=F$

Lemma 3.7

ind. hyp.

If $n>0$, then $\sum_{i=1}^{n} a_{i} . E_{i}+\sum_{j=1}^{m} W_{j} \stackrel{a_{n}}{\longrightarrow} E_{n}$. By $(A)$ and the soundness of the proof system, $\Sigma_{i=1}^{n} a_{i} . E_{i}+\sum_{j=1}^{m} W_{j}={ }_{b}^{\Delta} F$, it follows that $F \stackrel{a_{n}}{\longrightarrow} F^{\prime}$ with $F^{\prime} \approx_{b}^{\Delta} E_{n}$. Thus $\vdash F=F+a_{n} \cdot F^{\prime}$ follows from Lemma 3.7 Now

$\vdash \sum_{i=1}^{n} a_{i} . E_{i}+\sum_{j=1}^{m} W_{j}+F$

$=\sum_{i=1}^{n-1} a_{i} \cdot E_{i}+\sum_{j=1}^{m} W_{j}+F+a_{n} \cdot E_{n}$

$=\sum_{i=1}^{n-1} a_{i} \cdot E_{i}+\sum_{j=1}^{m} W_{j}+F+a_{n} \cdot \tau \cdot E_{n}$

$=\sum_{i=1}^{n-1} a_{i} \cdot E_{i}+\sum_{j=1}^{m} W_{j}+F+a_{n} \cdot \tau \cdot F^{\prime}$

$=\sum_{i=1}^{n-1} a_{i} \cdot E_{i}+\sum_{j=1}^{m} W_{j}+F+a_{n} \cdot F^{\prime}$

$=\sum_{i=1}^{n-1} a_{i} . E_{i}+\sum_{j=1}^{m} W_{j}+F$

$=F$

In the same way we can show $\vdash E+F=F$, hence

$\vdash E=E+F=F$.

\section{CONCLUSion AND Future Work}

In this paper we presented a complete axiomatisation for divergence-preserving branching congruence of finite-state behaviours. Also, along the way of proving soundness we identified three techniques for establishing divergence-preserving bisimulation equivalence and congruence: the $\mathcal{B}\left(\approx_{b}^{\Delta}\right)$ technique (proof of Lemma 2.12), the progressing branching bisimulation technique (proof of Lemma 3.3), and the strong bisimulation up to $\approx_{b}^{\Delta}$ technique (proof of Lemma 3.2). Since they help to relieve one off the burden of showing divergence preservation, these techniques enrich the theory of divergence-preserving branching bisimulation, and could be useful in other works. In [12] Aceto et al. studied complete axiomatisations for (divergence-blind) weak congruence, delay congruence, and $\eta$-congruence besides branching congruence. These other congruences also have corresponding divergencepreserving version similar to divergence-preserving branching congruence. We hope that the result of this paper may help to establish sound and complete axiomatisations for these divergence-preserving congruences.

\section{ACKNOWLEDGMENT}

The authors would like to thank David N. Jansen for proof reading a draft of the paper, and the anonymous referees for suggestions of improvement. The work has been supported by the CAS-INRIA major project No. GJHZ1844, and by NSFC under grants No. 61836005 and No. 62072443.

\section{REFERENCES}

[1] R. Milner, A Complete Inference System for a Class of Regular Behaviours, J. Comput. System Sci. 28, 439-466(1984).

[2] R. Milner, A Complete Axiomatisation for Observational Congruence of Finite-State Behaviours, Information and Computation 81, 227 247(1989).

[3] R. Milner, Communication and Concurrency, Pentice Hall 1995.

[4] D.J. Walker, Bisimulation and Divergence, Information and Computation 85, 202-241(1990).

[5] Rob J. van Glabbeek, The linear time-branching time spectrum I. The semantics of concrete, sequential processes, in: Handbook of Process Algebra, Elsevier, 2001, pp. 3-99(Chapter 1).

[6] Rob J. van Glabbeek, The linear time-branching time spectrum II. The semantics of sequential systems with silent moves (Extended abstract), in: Proc. CONCUR'93, LNCS vol. 715, Springer, 1993, pp.66-81.

[7] Jan Friso Groote and Frits Vaandrager, An Efficient Algorithm for Branching Bisimulation and Stuttering Equivalence, In the proceedings of the 17th International Colloquim on Automata, Languages and Programming, 1990 (ICALP1990).

[8] U. Montanari and V. Sassone, CCS Dynamic Bisimulation is Progressing, Proceedings of 16th international sumposium of Mathematical Foundations of Computer Science, 346-356 (1991).

[9] G. Winskel, A note on model checking the modal $\nu$-calculus, Theoretical Computer Science 83:157-167, 1991.

[10] Rob J. van Glabbeek, Peter Weijland: Branching time and abstraction in bisimulation semantics. J. ACM 43(3):555-600,1996.

[11] R.J. van Glabbeek, A Complete Axiomatisation for Branching Bisimulation Congruence of Finite-State Behaviours, MFCS 1996.

[12] L. Aceto, R. van Glabbeek, W. Fokkink, and A. Inglf́fsdóttir. Axiomatizing prefix iteration with silent steps. Information and Computation, 127(1):26-40, 1996.

[13] M. Lohrey, P.R. D'Argenio and H. Hermanns, Axiomatising Divergence, Information and Computation 203, 115-144(2005).

[14] T. Chen and J. Lu, Complete Axiomatisation for Divergence-Sensitive Bisimulations in Basic Process Algebra with Prefix Iteration, Electronic Notes in Theoretical Computer Scinece 212, 55-70(2008).

[15] Rob J. van Glabbeek, Bas Luttik, Nikola Trcka, Branching Bisimilarity with Explicit Divergence. Fundam. Inform. 93(4): 371-392, 2009.

[16] Yuxi Fu, Non-deterministic structure of computation, Math. Struct. Comput. Sci. 25(6): 1295-1338(2015)

[17] Xinxin Liu, Tingting Yu, and Wenhui Zhang, Analyzing divergence in bisimulation semantics. In proceedings of POPL 2017.

[18] Rob J. van Glavveek, Bas Luttik, Linda Spaninks, Rooted DivergencePreserving Branching Bisimilarity is a Congruence. Logical Methods in Computer Science, Volume 16, Issue 3, pp.14:1-14:16,2020.

[19] Clemens Grabmayer and Wan Fokkink, A Complete Proof System for 1Free Regular Expressions Modulo Bisimilarity. In the proceedings of 35th Annual Symposium on Logic in Computer Science, 2020, (LICS2020).

[20] Xinxin Liu and Tingting Yu, Canonical Solution to Recursive Equations and Completeness of Axiomatisations. In the roceedings of the international conference on Concurrency Theory 2020 (CONCUR2020). 